\title{
On the error term in Weyl's law for the Heisenberg manifolds
}

\author{
Wenguang ZHAI (Jinan)
}

\begin{abstract}
For a fixed integer $l \geq 1$, let $R(t)$ denote the error term in the Weyl's law of a $(2 l+1)$-dimensional Heisenberg manifold with the metric $g_{l}$. In this paper we shall prove the asymptotic formula of the $k$-th power moment for any integers $3 \leq k \leq 9$. We shall also prove that the function $t^{-(l-1 / 4)} R(t)$ has a distribution function.
\end{abstract}

\section{Contents}

$\S 1$. Introduction

1.1 The Weyl's law for $\mathbb{T}^{2}$ : the Gauss circle problem

1.2 The Weyl's law for 3-dimensional Heisenberg manifold

$\S 2$. Main Results

2.1 New results for power moments of $R(t)$

2.2 Idea of the proof

$\S 3$. Background of Heisenberg manifolds and the $\psi$-expression of $R(t)$

3.1 Heisenberg manifolds

3.2 The spectrum of Heisenberg manifolds

3.3 the $\psi$-expression of $R(t)$

3.4 A weighted lattice point problem

$\S 4$. Some preliminary Lemmas

$\S 5$. Proof of Theorem 1

5.1 Large value estimate of $R_{*}(x)$

5.2 Proof of Theorem 1

$\S 6$. Proof of Theorem 2

6.1 The upper bound of $\int_{T}^{2 T} G(x) d x$

6.2 An analogue of Voronoi's formula for $R_{*}(x)$

2000 Mathematics Subject Classification: 11N37, 35P20, 58J50.

Key Words: Heisenberg manifold, error term, exponential sum, power moment.

This work is supported by National Natural Science Foundation of China(Grant No. 10301018) and National Natural Science Foundation of Shandong Province(Grant No. 2006A31). 
6.3 Evaluation of the integral $\int_{T}^{2 T} F_{1}^{k}(x) d x$

6.4 Upper bound of the integral $\int_{T}^{2 T} F_{1}^{k-1}(x) F_{2}(x) d x$

6.5 Higher-power moments of $F_{2}(x)$

6.6 Evaluation of the integral $\int_{T}^{2 T} F^{k}(x) d x$

6.7 Proof of Theorem 2

$\S 7$. Proofs of Theorem 3 and Theorem 4

7.1 Proof of Theorem 3

7.2 Proof of Theorem 4(the case $k=3$ )

7.3 Proof of Theorem 4(the case $k=4$ )

$\S 8$. Proofs of Theorem 5 and Theorem 6

\section{Introduction}

Let $(M, g)$ be a closed $n$-dimensional Riemannian manifold with metric $g$ and Laplace-Beltrami operator $\Delta$. Let $N(t)$ denote its spectral counting function, which is defined as the number of the eigenvalues of $\Delta$ not exceeding $t$. Hörmander [13] proved that the Weyl's law

$$
N(t)=\frac{\operatorname{vol}\left(B_{n}\right) \operatorname{vol}(M)}{(2 \pi)^{n}} t^{n / 2}+O\left(t^{(n-1) / 2}\right)
$$

holds, where $\operatorname{vol}\left(B_{n}\right)$ is the volume of the $n$-dimensional unit ball.

Let

$$
R(t)=N(t)-\frac{\operatorname{vol}\left(B_{n}\right) \operatorname{vol}(M)}{(2 \pi)^{n}} t^{n / 2} .
$$

Hörmander's estimate (1.1) in general is sharp, as the well-known example of the sphere $S^{n}$ with its canonical metric shows [13]. However, it is a very difficult problem to determine the optimal bound of $R(t)$ in any given manifold, which depends on the properties of the associated geodesic flow. Many improvements have been obtained for certain types of manifolds, see [1, 2, 3, 4, 7, 9, 14, 16, 18, 24, 29].

\subsection{The Weyl's law for $\mathbb{T}^{2}$ : the Gauss circle problem}

The simplest compact manifold with integrable geodesic flow is the 2-torus $\mathbb{T}^{2}=\mathbb{R}^{2} / \mathbb{Z}^{2}$. The exponential functions $e(m x+n y)(m, n \in \mathbb{Z})$ form a basis of eigenfunctions of the Laplace operator $\Delta=\partial_{x}^{2}+\partial_{y}^{2}$, which acts on functions on $\mathbb{T}^{2}$. The corresponding eigenvalues are $4 \pi^{2}\left(m^{2}+n^{2}\right), m, n \in \mathbb{Z}$. The spectral counting function

$$
N_{I}(t)=\left\{\lambda_{j} \in \operatorname{Spec}(\Delta): \lambda_{j} \leq t\right\}
$$


is equal to the number of lattice points of $\mathbb{Z}^{2}$ inside a circle of radius $\sqrt{t} / 2 \pi$. The well-known Gauss circle problem is to study the properties of the error term of the function $N_{I}(t)$.

In this case, the formula (1.1) becomes

$$
N_{I}(t)=\frac{t}{4 \pi}+O\left(t^{1 / 2}\right),
$$

which is the classical result of Gauss. Let $R_{I}(t)$ denote the error term in (1.2). Many authors improved the upper bound estimate of $R_{I}(t)$. The latest result is due to Huxley[14], which reads

$$
R_{I}(t) \ll t^{131 / 416} \log ^{26957 / 8320} t .
$$

Hardy [10] conjectured that

$$
R_{I}(t) \ll t^{1 / 4+\varepsilon}
$$

Cramér [5] proved that

$$
\lim _{T \rightarrow \infty} T^{-3 / 2} \int_{1}^{T}\left|R_{I}(t)\right|^{2} d t=C, \quad C=\frac{1}{6 \pi^{3}} \sum_{n=1}^{\infty} \frac{r^{2}(n)}{n^{3 / 2}},
$$

which is consistent with Hardy's conjecture, where $r(n)$ denotes the number of ways $n$ can be written as a sum of two squares.

Ivić[15] first used the large value technique to study the higher power moments of $R_{I}(t)$. He proved that the estimate

$$
\int_{1}^{T}\left|R_{I}(t)\right|^{A} d t \ll T^{1+A / 4+\varepsilon}
$$

holds for each fixed $0 \leq A \leq 35 / 8$. The value of $A$ for which (1.5) holds is closely related to the upper bound of $R_{I}(t)$. If we insert the estimate (1.3) into Ivić's machinery, we get that (1.5) holds for $0 \leq A \leq 262 / 27$.

Tsang [26] studied the third and the fourth moments of $R_{I}(t)$. He proved that the following two asymptotic formulas are true,

$$
\begin{aligned}
& \int_{1}^{T} R_{I}^{3}(t) d t=c_{3} T^{7 / 4}+O\left(T^{7 / 4-1 / 14+\varepsilon}\right) \\
& \int_{1}^{T} R_{I}^{4}(t) d t=c_{4} T^{2}+O\left(T^{2-1 / 23+\varepsilon}\right)
\end{aligned}
$$


where $c_{3}$ and $c_{4}$ are explicit constants.

Heath-Brown[11] proved that the function $t^{-1 / 4} R_{I}(t)$ has a distribution function $f(\alpha)$ in the sense that, for any interval $I$ we have

$$
T^{-1} \operatorname{mes}\left\{t \in[1, T]: t^{-1 / 4} R_{I}(t) \in I\right\} \rightarrow \int_{I} f(\alpha) d \alpha
$$

as $T \rightarrow \infty$. He also proved that for any real number $k \in[0,9]$ the mean value

$$
\lim _{T \rightarrow \infty} T^{-1-k / 4} \int_{1}^{T}\left|R_{I}(t)\right|^{k} d t
$$

converges to a finite limit as $T$ tends to infinity. Moreover the same is true for

$$
\lim _{T \rightarrow \infty} T^{-1-k / 4} \int_{1}^{T} R_{I}(t)^{k} d t
$$

with $k=3,5,7,9$.

In [30], the author proved the following result: let $A>9$ be a real number such that (1.5) holds, then for any integer $3 \leq k<A$, we have the asymptotic formula

$$
\int_{1}^{T} R_{I}^{k}(t) d t=c_{k} T^{1+k / 4}+O\left(T^{1+k / 4-\delta_{k}+\varepsilon}\right),
$$

where $c_{k}$ and $\delta_{k}>0$ are explicit constants. Especially, the asymptotic formula (1.8) holds for $k=3,4,5,6,7,8,9$.

We remark that for $\delta_{3}$ we can take $\delta_{3}=7 / 20$, which is due to Tsang for the third moment of the error term of the Dirichlet divisor problem. However, Tsang didn't publish this result.

For the fourth power moment of $R_{I}(t)$, the author 31 proved that we can take $\delta_{4}=3 / 28$.

\subsection{The Weyl's law for $(2 l+1)$-dimensional Heisenberg man- ifold}

Let $l \geq 1$ be a fixed integer and $\left(H_{l} / \Gamma, g\right)$ be a $(2 l+1)$-dimensional Heisenberg manifold with a metric $g$. When $l=1$, in 24 Petridis and Toth proved that $R(t)=O\left(t^{5 / 6} \log t\right)$ for a special metric. Later in 4 this bound was improved to $O\left(t^{119 / 146+\varepsilon}\right)$ for all left-invariant Heisenberg metrics. For $l>1$ Khosravi and Petridis 18 proved that $R(t)=O\left(t^{l-7 / 41}\right)$ holds for rational Heisenberg manifolds. 
Both in [4] and [18, they first established a $\psi$-expression of $R(t)$ and then used the van der Corput method of exponential sums . Substituting Huxley's result of [14] into the arguments of [4] and [18], we can get that the estimate

$$
R(t)=O\left(t^{l-77 / 416}(\log t)^{26957 / 8320}\right)
$$

holds for all rational $(2 l+1)$-dimensional Heisenberg manifolds.

It was conjectured that for rational Heisenberg manifolds, the pointwise estimate

$$
R(t) \ll t^{l-1 / 4+\varepsilon}
$$

holds, which was proposed in Petridis and Toth [24] for the case $l=1$ and in Khosravi and Petridis[18] for the case $l>1$. As an evidence of this conjecture, Petridis and Toth proved the following $L^{2}$ result for $H_{1}$

$$
\int_{I^{3}}\left|N(t ; u)-\frac{1}{6 \pi^{2}} \operatorname{vol}(M(u)) t^{3 / 2}\right|^{2} d u \leq C_{\delta} t^{3 / 2+\delta},
$$

where $I=[1-\varepsilon, 1+\varepsilon]$. They also proved

$$
\frac{1}{T} \int_{T}^{2 T}\left|N(t)-\frac{1}{6 \pi^{2}} \operatorname{vol}(M) t^{3 / 2}\right| d t \gg T^{3 / 4}
$$

Now let $M=\left(H_{l} / \Gamma, g_{l}\right)$ be a $(2 l+1)$-dimensional Heisenberg manifold with the metric

$$
g_{l}:=\left(\begin{array}{ll}
I_{2 l \times 2 l} & 0 \\
0 & 2 \pi
\end{array}\right),
$$

where $I_{2 l \times 2 l}$ is the identity matrix

M. Khosravi and John A. Toth 19 proved that

$$
\int_{1}^{T}|R(t)|^{2} d t=C_{2, l} T^{2 l+1 / 2}+O\left(T^{2 l+1 / 4+\varepsilon}\right),
$$

where $C_{2, l}$ is an explicit constant .

M. Khosravi [17] proved that the asymptotic formula

$$
\int_{1}^{T} R^{3}(t) d t=C_{3, l} T^{3 l+1 / 4}+O\left(T^{3 l+3 / 14+\varepsilon}\right)
$$

is true for some explicit constant $C_{3, l}$.

The aim of this paper is to prove some power moment results for $R(t)$, which are analogous to the results of $R_{I}(t)$ stated in Section 1.1. The plan of this paper 
is as follows. In Section 2 we shall state our main results. In Section 3 we state some background of the Heisenberg manifolds and give a new $\psi$-expression of $R(t)$. In Section 4 we quote some preliminary Lemmas. We shall prove our theorems in Section 5-7.

Notations. For a real number $t$, let $[t]$ denote the integer part of $t,\{t\}=t-[t]$, $\psi(t)=\{t\}-1 / 2,\|t\|=\min (\{t\}, 1-\{t\}), e(t)=e^{2 \pi i t} . \varepsilon$ always denotes a sufficiently small positive constant. $\mathbb{C}, \mathbb{R}, \mathbb{Z}, \mathbb{N}$ denote the set of complex numbers, the set of real numbers, the set of integers, the set of positive integers, respectively. $n \sim M$ means that $N<n \leq 2 N . d(n)$ denotes the Dirichlet divisor function, $r(n)$ denotes the number of ways $n$ can be written as a sum of two squares, $\mu(n)$ denotes the Möbius function. Throughout this paper, $\mathcal{L}$ always denotes $\log T$.

\section{Main results}

From now on, we always suppose that $R(t)$ denote the error term in the Weyl's law for the $(2 l+1)$-dimensional Heisenberg manifold $\left(H_{l} / \Gamma, g_{l}\right)$.

\subsection{New results for power moments of $R(t)$}

Our first result is the following Theorem 1, which is an analogue of (1.5) for $R_{I}(t)$.

Theorem 1. Suppose $A \geq 0$ is a fixed real number. Then

$$
\begin{aligned}
& \int_{1}^{T}|R(t)|^{A} d t \ll T^{1+A(l-1 / 4)} \mathcal{L}^{41} \quad(0 \leq A \leq 262 / 27), \\
& \int_{1}^{T}|R(t)|^{A} d t \ll T^{A(l-1)+\frac{154+339 A}{416}} \mathcal{L}^{4 A+1} \quad(A>262 / 27) .
\end{aligned}
$$

Remark 2.1. Let $A_{0}>1$ be a positive constant such that the estimate

$$
\int_{1}^{T}|R(t)|^{A_{0}} d t \ll T^{1+A_{0}(l-1 / 4)+\varepsilon}
$$

holds, then Theorem 1 states that we can take $A_{0}=262 / 27$. The value of $A_{0}$ for which (2.3) holds is closely related to the upper bound estimate of $R(t)$. The exponent 262/27 follows from the estimate (1.9). If the conjecture (1.10) were true, then obviously (2.3) were true for any $A_{0}>0$. Conversely, if the estimate (2.3) were 
true for any $A_{0}>0$, then we could show that the estimate (1.10) were also true. The argument is as follows. The estimate (2.3) implies that the upper bound

$$
\int_{T / 2}^{T}|R(2 \pi x)|^{A_{0}} d x \ll T^{1+A_{0}(l-1 / 4)+\varepsilon} .
$$

Suppose $|R(2 \pi x)|$ reaches its largest value $V_{0}$ at some $x_{0} \in[T / 2, T]$. According to the formula (5.11), we have

$$
V_{0}^{A_{0}+1}\left(x_{0}^{l-1 / 2} \log x_{0}\right)^{-1} \ll \int_{T / 2}^{T}|R(2 \pi x)|^{A_{0}} d x \ll T^{1+A_{0}(l-1 / 4)+\varepsilon},
$$

which implies that

$$
V_{0} \ll T^{\frac{l+1 / 2+A_{0}(l-1 / 4)+2 \varepsilon}{\left(A_{0}+1\right)}} .
$$

Now the conjecture (1.10) follows from the above estimate by choosing $A_{0}$ large.

Before stating our next theorem, we introduce some notations.

Suppose $f: \mathbb{N} \rightarrow \mathbb{R}$ is any function such that $f(n) \ll n^{\varepsilon}, k \geq 2$ is a fixed integer. Define

$$
\begin{gathered}
s_{k ; v}(f):=\sum_{\sqrt{n_{1}}+\cdots+\sqrt{n_{v}}=\sqrt{n_{v+1}}+\cdots+\sqrt{n_{k}}} \frac{f\left(n_{1}\right) \cdots f\left(n_{k}\right)}{\left(n_{1} \cdots n_{k}\right)^{3 / 4}}(1 \leq v<k), \\
B_{k}(f):=\sum_{v=1}^{k-1}\left(\begin{array}{c}
k-1 \\
v
\end{array}\right) s_{k ; v}(f) \cos \frac{\pi(k-2 v)}{4}, \\
\tau_{l}(n):=\sum_{n=h(2 r-h)} \frac{e(l h / 2) h^{1 / 2}}{(2 r-h)^{1 / 2}}\left(1-\frac{h}{2 r-h}\right)^{l-1} .
\end{gathered}
$$

We shall use $s_{k ; v}(f)$ to denote both of the series (2.4) and its value. The convergence of $s_{k ; v}(f)$ was already proved in the author [30]. It is obvious that $\left|\tau_{l}(n)\right| \leq d(n)$.

Suppose $A_{0}>2$ is a real number, define

$$
\begin{aligned}
& K_{0}:=\min \left\{n \in \mathbb{N}: n \geq A_{0}, 2 \mid n\right\}, \\
& s(k):=2^{k-2}+(k-6) / 4, \\
& \sigma\left(k, A_{0}\right):=\frac{A_{0}-k}{2\left(A_{0}-2\right)}, \\
& \delta_{1}\left(k, A_{0}\right):=\sigma\left(k, A_{0}\right) / 2 s\left(K_{0}\right), \\
& \delta_{2}\left(k, A_{0}\right):=\frac{\sigma\left(k, A_{0}\right)}{2 s(k)+2 \sigma\left(k, A_{0}\right)} .
\end{aligned}
$$


Theorem 2. Let $A_{0}>9$ be a real number such that (2.3) holds, then for any integer $3 \leq k<A_{0}$, we have the asymptotic formula

$$
\int_{1}^{T} R^{k}(t) d t=\frac{2^{3+5 k / 4-2 k l} l^{k} B_{k}\left(\tau_{l}\right)}{(l !)^{k} \pi^{3 k / 4+k l}(4+k(4 l-1))} T^{1+k(l-1 / 4)}+O\left(T^{1+k(l-1 / 4)-\delta_{1}\left(k, A_{0}\right)+\varepsilon}\right) .
$$

Remark 2.2. From Theorem 1 we see that for $k \in\{3,4,5,6,7,8,9\}$, we can get the asymptotic formula (2.7) with $A_{0}=262 / 27$. Moreover, the asymptotic (2.7) provides the exact form of the main term for the integral $\int_{1}^{T} R^{k}(t) d t$ for $k \geq 10$. From Theorem 2 we see that if the conjecture (1.10) were true, then for all $k \geq 3$ we could get the asymptotic formula of $\int_{1}^{T} R^{k}(t) d t$.

When $3 \leq k \leq 9$, we have the following Theorem 3, which is better than Theorem 2.

Theorem 3. For $3 \leq k \leq 9$, the asymptotic formula (2.7) holds with $\delta_{1}\left(k, A_{0}\right)$ replaced by $\delta_{2}(k, 262 / 27)$.

Remark 2.3. Note that $\delta_{2}(3,262 / 27)=181 / 1402$ and $\delta_{2}(4,262 / 27)=11 / 230$, which are small when comparison to the corresponding results of $R_{I}(t)$. The following Theorem 4 improves these two exponents.

Theorem 4. We have

$$
\int_{1}^{T} R^{3}(t) d t=\frac{2^{27 / 4-6 l} l^{3} B_{3}\left(\tau_{l}\right)}{(l !)^{3} \pi^{3 l+9 / 4}(1+12 l)} T^{3 l+1 / 4}+O\left(T^{3 l+\varepsilon}\right)
$$

and

$$
\int_{1}^{T} R^{4}(t) d t=\frac{2^{4-8 l} l^{3} B_{4}\left(\tau_{l}\right)}{(l !)^{4} \pi^{4 l+3}} T^{4 l}+O\left(T^{4 l-3 / 28+\varepsilon}\right) .
$$

The following two theorems are analogous to Heath-Brown's results for $R_{I}(t)$.

Theorem 5. The function $t^{-(l-1 / 4)} R(t)$ has a distribution function $f(\alpha)$ in the sense that, for any interval $I \subset \mathbb{R}$ we have

$$
T^{-1} \operatorname{mes}\left\{t \in[1, T]: t^{-(l-1 / 4)} R(t) \in I\right\} \rightarrow \int_{I} f(\alpha) d \alpha
$$

as $T \rightarrow \infty$. The function $f(\alpha)$ and its derivatives satisfy

$$
\frac{d^{k}}{d \alpha^{k}} f(\alpha) \ll_{A, k}(1+|\alpha|)^{-A}
$$

for $k=0,1,2, \cdots$ and $f(\alpha)$ can be extended to an entire function. 
Theorem 6. For any real number $k \in[0,262 / 27)$ the mean value

$$
\lim _{T \rightarrow \infty} T^{-1-k(l-1 / 4)} \int_{1}^{T}|R(t)|^{k} d t
$$

converges to a finite limit as $T$ tends to infinity.

Remark 2.4. Our approach of this paper implies a different proof of the formula (1.11) and the error term estimate $O\left(T^{2 l+1 / 4+\varepsilon}\right)$ therein can be improved slightly to $O\left(T^{2 l+1 / 4} \mathcal{L}^{3}\right)$. However, it is difficult to improve the exponent $2 l+1 / 4$. For the constant $C_{2, l}$ contained in (1.11), we have a new expression, namely,

$$
C_{2, l}=\frac{2^{9 / 2-4 l} l^{2} B_{2}\left(\tau_{l}\right)}{(l !)^{2} \pi^{2 l+3 / 2}(4 l+1)}=\frac{2^{9 / 2-4 l} l^{2}}{(l !)^{2} \pi^{2 l+3 / 2}(4 l+1)} \sum_{n=1}^{\infty} \frac{\tau_{l}^{2}(n)}{n^{3 / 2}},
$$

which is analogous to the expression of $C$ in Cramér's result for the mean square of $R_{I}(t)$.

\section{$2.2 \quad$ Idea of the proof}

M. Khosvari and John A. Toth[19] introduced an extra parameter and then used the Poisson summation formula to write the corresponding error term in a form which can be estimated by the method of the stationary phase. Finally they eliminated the parameter and got the asymptotic formula (1.11). This approach worked very well for the mean square of $R(t)$. M. Khosvari[17] used the same approach to the third moment of $R(t)$ and proved (1.12). But it is difficult to use this approach to the $k$-th power moment when $k \geq 4$.

In this paper we shall use a different approach to prove our theorems. We can establish an analogy between $R_{I}(t)$ and $R(t)$ and then we prove our theorems by using this analogy.

It is well-known that $R_{I}(t)$ has the following truncated Voronoi's formula

$$
R_{I}(t)=-\frac{1}{2^{1 / 2} \pi^{3 / 2}} \sum_{n \leq N} r(n) n^{-3 / 4} t^{1 / 4} \cos (2 \sqrt{n t}+\pi / 4)+O\left(t^{1 / 2+\varepsilon} N^{-1 / 2}\right)
$$

for $1 \leq N \ll t$, which follows from Lemma 3 of Müller[22]. The formula (2.10) plays an essential role in the proofs of all results stated in Section 1.1.

$R(t)$ is a sum involving the row-teeth-function $\psi(u)$ and doesn't have such a direct and easily usable Voronoi's formula at hand. However, by using the finite expression of $\psi(u)$ (see Lemma 4.2)and van der Corput's B-process(see Lemma 4.3) 
we can prove an formula(see Proposition 6.1) analogous to (2.10). This formula, although is weak when comparison to (2.10), is enough to prove all our results.

Nowak 23] and Kuhleitner, Nowak 20] first studied mean squares of error terms involving $\psi(u)$, where they used another finite Fourier expansion of $\psi(u)$, which is a much more precise form than Lemma 4.1. However, in our paper, we only use Lemma 4.1 to study the upper bound of $R(t)$. For the asymptotic results of $R(t)$, we shall use Lema 4.2, which seems more convenient to use. The method of this paper can be used to study the power moments of other error terms involving the function $\psi(u)$.

\section{Background of Heisenberg manifolds and the $\psi$-expression of $R(t)$}

In this section, we first review some background of the Heisenberg manifolds. The reader can see [6], 8] , 25] for more details. Finally, we give an $\psi$-expression of $R(t)$.

\subsection{Heisenberg manifolds}

Suppose $x \in \mathbb{R}^{l}$ is a row vector and $y \in \mathbb{R}^{l}$ is a column vector. Define

$$
\gamma(x, y, t)=\left(\begin{array}{lll}
1 & x & t \\
0 & I_{l} & y \\
0 & 0 & 1
\end{array}\right), \quad X(x, y, t)=\left(\begin{array}{lll}
0 & x & t \\
0 & 0 & y \\
0 & 0 & 0
\end{array}\right) \text {. }
$$

The $(2 l+1)$-dimensional Heisenberg group $H_{l}$ is defined by

$$
H_{l}=\left\{\gamma(x, y, t): x, y \in \mathbb{R}^{l}, t \in \mathbb{R}\right\},
$$

its Lie algebra is

$$
\mathfrak{H}_{l}=\left\{X(x, y, t): x, y \in \mathbb{R}^{l}, t \in \mathbb{R}\right\} .
$$

We say $\Gamma$ is uniform discrete subgroup of $H_{l}$ if $H_{l} / \Gamma$ is compact. A $(2 l+1)$ dimensional Heisenberg manifold is a pair $\left(H_{l} / \Gamma, g\right)$ for which $\Gamma$ is a uniform discrete subgroup of $H_{l}$ and $g$ is a left $H_{l}$-invariant metric.

For every $r$-tuple $\left(r_{1}, r_{2}, \cdots, r_{l}\right) \in \mathbb{N}^{l}$ such that $r_{j} \mid r_{j+1}(j=1,2, \cdots, l-1)$, let $r \mathbb{Z}^{l}$ denote the $l$-tuples $x=\left(x_{1}, x_{2}, \cdots, x_{l}\right)$ with $x_{j} \in r_{j} \mathbb{Z}$. Define

$$
\Gamma_{r}=\left\{\gamma(x, y, t): x \in r \mathbb{Z}^{l}, y \in r \mathbb{Z}^{l}, t \in \mathbb{Z}\right\} .
$$


It is clear that $\Gamma_{r}$ is a uniform discrete subgroup of $H_{l}$. According to Theorem 2.4 of [8], the subgroup $\Gamma_{r}$ classifies all the uniform discrete subgroups of $H_{l}$ up to automorphisms. Thus (see [8], Corollary 2.5) given any Riemannian Heisenberg manifold $M=\left(H_{l} / \Gamma, g\right)$, there exists a unique $l$-tuple $r$ as before and a left-invariant metric $\tilde{g}$ on $H_{l}$ such that $M$ is isometric to $\left(H_{l} / \Gamma, \tilde{g}\right)$. So (see [8], 2.6(b)) we can replace the metric $g$ by $\phi^{*} g$, where $\phi$ is an inner automorphism such that the direct sum split of the Lie algebra $\mathfrak{H}_{l}=\mathbb{R}^{2 l} \oplus \mathfrak{Z}$ is orthogonal . Here $\mathfrak{Z}$ is the center of the Lie algebra and

$$
\mathbb{R}^{2 l}=\left\{\left(\begin{array}{lll}
0 & x & 0 \\
0 & 0 & y \\
0 & 0 & 0
\end{array}\right): x, y \in \mathbb{R}^{l}\right\} .
$$

With respect to this orthogonal split of $H_{l}$ the metric $g$ has the form

$$
\left(\begin{array}{ll}
h & 0 \\
0 & g_{2 l+1}
\end{array}\right)
$$

where $h$ is a positive-definite $2 l \times 2 l$ matrix and $g_{2 l+1}>0$ is a real number.

The volume of the Heisenberg manifold is given by

$$
\operatorname{vol}\left(H_{l} / \Gamma, g\right)=\left|\Gamma_{r}\right| \sqrt{\operatorname{det}(g)}
$$

with $\left|\Gamma_{r}\right|=r_{1} r_{2} \cdots r_{l}$ for $r=\left(r_{1}, r_{2}, \cdots, r_{l}\right)$.

\subsection{The spectrum of Heisenberg manifolds}

Let $\Sigma$ be the spectrum of the Laplacian on $M=\left(H_{l} / \Gamma, g_{l}\right)$, where the eigenvalues are counted with multiplicities. According to [8](P. 258), $\Sigma$ can be divided into two parts $\Sigma_{1}$ and $\Sigma_{2}$, where $\Sigma_{1}$ is the spectrum of $2 l$-dimensional torus and $\Sigma_{2}$ contains all eigenvalues of the form

$$
2 \pi m^{2}+2 \pi m\left(2 n_{1}+\cdots+2 n_{l}+l\right), m \in \mathbb{N}, n_{j} \in \mathbb{N} \cup\{0\},
$$

each eigenvalue counted with the multiplicity $2 m^{l}$.

\section{3 the $\psi$-expression of $R(t)$}

In this section we shall prove the following $\psi$-expression of $R(t)$. 
Lemma 3.1. We have

$$
R(2 \pi x)=-\frac{4}{2^{l}(l-1) !} \sum_{1 \leq m \leq \sqrt{x}} m\left(x-m^{2}\right)^{l-1} \psi\left(\frac{x}{2 m}-\frac{m}{2}-\frac{l}{2}\right)+O\left(x^{l-1 / 2}\right) .
$$

Let

$$
N(t)=N_{I}(t)+N_{I I}(t)
$$

where

$$
\begin{aligned}
& N_{I}(t):=\#\left\{\lambda: \lambda \in \Sigma_{1}, \lambda \leq t\right\}, \\
& N_{I I}(t):=\#\left\{\lambda: \lambda \in \Sigma_{2}, \lambda \leq t\right\} .
\end{aligned}
$$

For $N_{I}(t)$ by Hörmander's theorem we have

$$
N_{I}(t)=\frac{1}{l ! 2^{l}}\left(\frac{t}{2 \pi}\right)^{l}+O\left(t^{l-1 / 2}\right) .
$$

\subsubsection{Proof of Lemma 3.1 for $l=1$}

Suppose $l=1$. It is easily seen that

$$
N_{I I}(t)=\sum_{\substack{m^{2}+m(2 n+1) \leq t / 2 \pi \\ m \geq 1, n \geq 0}} 2 m
$$

Thus we get

$$
\begin{aligned}
N_{I I}(2 \pi x) & =\sum_{\substack{m^{2}+m(2 n+1) \leq x \\
m \geq 1, n \geq 0}} 2 m=\sum_{\substack{m^{2}+m(2 n+1) \leq x \\
1 \leq m \leq \sqrt{x}, n \geq 0}} 2 m+O(\sqrt{x}) \\
& =\sum_{1 \leq m \leq \sqrt{x}} 2 m \sum_{\substack{0 \leq n \leq \frac{x}{2 m}-\frac{m}{2}-\frac{1}{2} \\
+}} 2 m(\sqrt{x}) \\
& =\sum_{1 \leq m \leq \sqrt{x}} 2 m\left[\frac{x}{2 m}-\frac{m}{2}+\frac{1}{2}\right]+O(\sqrt{x}) \\
& =\sum_{1 \leq m \leq \sqrt{x}} 2 m\left(\frac{x}{2 m}-\frac{m}{2}\right)-\sum_{1 \leq m \leq \sqrt{x}} 2 m \psi\left(\frac{x}{2 m}-\frac{m}{2}+\frac{1}{2}\right)+O(\sqrt{x}) \\
& =\frac{2}{3} x^{3 / 2}-\frac{x}{2}-\sum_{1 \leq m \leq \sqrt{x}} 2 m \psi\left(\frac{x}{2 m}-\frac{m}{2}+\frac{1}{2}\right)+O(\sqrt{x}),
\end{aligned}
$$

which combined with (3.3) for $l=1$ proves Lemma 3.1 of the case $l=1$. 


\subsubsection{Proof of Lemma 3.1 for $l>1$}

Suppose now $l>1$. In this case for $N_{I I}(t)$ we have

$$
\begin{aligned}
N_{I I}(t) & =\sum_{\substack{m^{2}+m\left(2 n_{1}+\cdots+2 n_{l}+l\right) \leq t / 2 \pi \\
m>0, n_{j} \geq 0}} 2 m^{l} \\
& =\sum_{\substack{m^{2}+m(2 n+l) \leq t / 2 \pi \\
m>0, n \geq 0}} 2 m^{l} \sum_{\substack{n=n_{1}+\cdots+n_{l} \\
n_{j} \geq 0}} 1 \\
= & \sum_{\substack{m^{2}+m(2 n+l) \leq t / 2 \pi \\
m>0, n \geq 0}} 2 m^{l}\left(\begin{array}{c}
n+l-1 \\
l-1
\end{array}\right) \\
= & \frac{2}{(l-1) !} \sum_{m^{2}+m(2 n+l) \leq t / 2 \pi}^{m>0, n \geq 0} m^{l} n^{l-1} \\
& +\frac{l}{(l-2) !} \sum_{\substack{m^{2}+m(2 n+l) \leq t / 2 \pi \\
m>0, n \geq 0}} m^{l} n^{l-2}+O\left(t^{l-1 / 2}\right) . \\
= & \Sigma_{1, l}(t)+\Sigma_{2, l}(t)+O\left(t^{l-1 / 2}\right),
\end{aligned}
$$

say.

In order to evaluate $\Sigma_{1, l}(t)$ and $\Sigma_{2, l}(t)$, we need the following

Lemma 3.2. (Euler-Maclaurin summation formula) Suppose that $f \in C^{1}[a, y]$, $a \in \mathbb{Z}$, then

$$
\sum_{a \leq n \leq y} f(n)=\int_{a}^{y}\left(f(t)+\psi(t) f^{\prime}(t)\right) d t-\psi(y) f(y)+\frac{f(a)}{2} .
$$

Suppose $d \geq 0$ is a fixed integer. By Lemma 3.2 we get

$$
\sum_{0 \leq n \leq y} n^{d}= \begin{cases}y-\psi(y)+1 / 2, & \text { if } d=0 \\ \frac{y^{d+1}}{d+1}-\psi(y) y^{d}+O\left(y^{d-1}\right), & \text { if } d \geq 1\end{cases}
$$


By (3.6) we get

$$
\text { (3.7) } \begin{aligned}
\Sigma_{1, l}(2 \pi x) & =\frac{2}{l !} \sum_{1 \leq m \leq \sqrt{x}} m^{l}\left(\frac{x}{2 m}-\frac{m}{2}-\frac{l}{2}\right)^{l} \\
& -\frac{2}{(l-1) !} \sum_{1 \leq m \leq \sqrt{x}} m^{l}\left(\frac{x}{2 m}-\frac{m}{2}-\frac{l}{2}\right)^{l-1} \psi\left(\frac{x}{2 m}-\frac{m}{2}-\frac{l}{2}\right) \\
& +O\left(x^{l-1 / 2}\right)
\end{aligned}
$$

and

(3.8) $\quad \Sigma_{2, l}(2 \pi x)=\frac{l}{(l-1) !} \sum_{1 \leq m \leq \sqrt{x}} m^{l}\left(\frac{x}{2 m}-\frac{m}{2}-\frac{l}{2}\right)^{l-1}+O\left(x^{l-1 / 2}\right)$.

Write

$$
\sum_{1 \leq m \leq \sqrt{x}} m^{l}\left(\frac{x}{2 m}-\frac{m}{2}-\frac{l}{2}\right)^{l}=2^{-l} \sum_{0 \leq m \leq \sqrt{x}}\left(x-m^{2}-m l\right)^{l}-2^{-l} x^{l} .
$$

Let $u(t)=\left(x-t^{2}-t l\right)^{l}$. It is easy to check that

$$
\begin{aligned}
& \int_{0}^{\sqrt{x}} u(t) d t=\frac{(l !)^{2} 2^{2 l}}{(2 l+1) !} x^{l+1 / 2}-\frac{l}{2} x^{l}+O\left(x^{l-1 / 2}\right), \\
& \int_{0}^{\sqrt{x}} \psi(t) u^{\prime}(t) d t \ll x^{l-1 / 2} .
\end{aligned}
$$

By Lemma 3.2 we have

$$
\begin{aligned}
& \sum_{0 \leq m \leq \sqrt{x}}\left(x-m^{2}-m l\right)^{l} \\
& =\int_{0}^{\sqrt{x}}\left(u(t)+\psi(t) u^{\prime}(t)\right) d t-\psi(\sqrt{x}) u(\sqrt{x})+\frac{u(0)}{2} \\
& =\frac{(l !)^{2} 2^{2 l}}{(2 l+1) !} x^{l+1 / 2}-\frac{l-1}{2} x^{l}+O\left(x^{l-1 / 2}\right) .
\end{aligned}
$$

From (3.9) and (3.10) we get

$$
\sum_{1 \leq m \leq \sqrt{x}} m^{l}\left(\frac{x}{2 m}-\frac{m}{2}-\frac{l}{2}\right)^{l}=\frac{(l !)^{2} 2^{l}}{(2 l+1) !} x^{l+1 / 2}-\frac{l+1}{2^{l+1}} x^{l}+O\left(x^{l-1 / 2}\right) .
$$


Write

$$
\sum_{1 \leq m \leq \sqrt{x}} m^{l}\left(\frac{x}{2 m}-\frac{m}{2}-\frac{l}{2}\right)^{l-1}=2^{-l+1} \sum_{1 \leq m \leq \sqrt{x}} m\left(x-m^{2}-m l\right)^{l-1} .
$$

Let $v(t)=t\left(x-t^{2}-t l\right)^{l-1}$. It is easy to check that

$$
\begin{aligned}
& \int_{1}^{\sqrt{x}} v(t) d t=\frac{1}{2 l} x^{l}+O\left(x^{l-1 / 2}\right), \\
& \int_{1}^{\sqrt{x}} \psi(t) v^{\prime}(t) d t \ll x^{l-1} .
\end{aligned}
$$

By Lemma 3.2 we have

$$
\begin{aligned}
& \sum_{1 \leq m \leq \sqrt{x}} m\left(x-m^{2}-m l\right)^{l-1} \\
= & \int_{1}^{\sqrt{x}}\left(v(t)+\psi(t) v^{\prime}(t)\right) d t-\psi(\sqrt{x}) v(\sqrt{x})+\frac{v(1)}{2} \\
= & \frac{1}{2 l} x^{l}+O\left(x^{l-1 / 2}\right) .
\end{aligned}
$$

From (3.12) and (3.13) we get

$$
\sum_{1 \leq m \leq \sqrt{x}} m^{l}\left(\frac{x}{2 m}-\frac{m}{2}-\frac{l}{2}\right)^{l-1}=\frac{1}{2^{l} l} x^{l}+O\left(x^{l-1 / 2}\right) .
$$

Since

$$
\left(\frac{x}{2 m}-\frac{m}{2}-\frac{l}{2}\right)^{l-1}-\left(\frac{x}{2 m}-\frac{m}{2}\right)^{l-1} \ll\left(\frac{x}{m}\right)^{l-2}
$$

holds for $m \ll \sqrt{x}$, we have

$$
\begin{aligned}
& \sum_{m^{2}+m l \leq x} m^{l}\left(\frac{x}{2 m}-\frac{m}{2}-\frac{l}{2}\right)^{l-1} \psi\left(\frac{x}{2 m}-\frac{m}{2}-\frac{l}{2}\right) \\
= & \sum_{m^{2}+m l \leq x} m^{l}\left(\frac{x}{2 m}-\frac{m}{2}\right)^{l-1} \psi\left(\frac{x}{2 m}-\frac{m}{2}-\frac{l}{2}\right)+O\left(x^{l-1 / 2}\right) . \\
= & \sum_{1 \leq m \leq \sqrt{x}} m^{l}\left(\frac{x}{2 m}-\frac{m}{2}\right)^{l-1} \psi\left(\frac{x}{2 m}-\frac{m}{2}-\frac{l}{2}\right)+O\left(x^{l-1 / 2}\right) .
\end{aligned}
$$

Now Lemma 3.1 follows from (3.3), (3.5), (3.7), (3.8), (3.11), (3.14) and (3.15) . 


\subsection{A weighted lattice point problem}

For any positive integer $d$, define

$$
f_{R}(d):=\sum_{\substack{d=m(m+2 n+l) \\ m>0, n \geq 0}} m^{l} n^{l-1} .
$$

From the proof of Lemma 3.1 it is easy to see that

$$
R(2 \pi x)=\frac{2}{(l-1) !} \sum_{d \leq x} f_{R}(d)-\frac{2^{l+1} l !}{(2 l+1) !} x^{l+1 / 2}-\frac{l+1}{2^{l} l !} x^{l}+O\left(x^{l-1 / 2}\right) .
$$

So the evaluation of the counting function $N(2 \pi x)$, is equivalent to study the asymptotic behavior of the mean value $\sum_{d \leq x} f_{R}(d)$.

\section{Some preliminary Lemmas}

We need the following Lemmas. Lemma 4.1 is due to Vaaler[27]. Lemma 4.2 is wellknown; see for example, Heath-Brown[12. Lemma 4.3 is Theorem 2.2 of Min[21, see also Lemma 6 of Chapter 1 in [28]. A weaker version of Lemma 4.3 can be found in [20], which also suffices for our proof. Lemma 4.4 is Lemma 3.1 of the author [30]. Lemma 4.5 is the first derivative test. Lemma 4.6 is the famous Halász-Montgomery inequality, see for example, Ivić[15].

Lemma 4.1. Let $H \geq 2$ be any real number. Then

$$
\psi(u)=\sum_{1 \leq|h| \leq H} a(h) e(h u)+O\left(\sum_{0 \leq|h| \leq H} b(h) e(h u)\right),
$$

where $a(h)$ and $b(h)$ are functions such that $a(h) \ll 1 /|h|, b(h) \ll 1 / H$.

Lemma 4.2. Let $H \geq 2$ be any real number. Then

$$
\psi(u)=-\sum_{1 \leq|h| \leq H} \frac{e(h u)}{2 \pi i h}+O\left(\min \left(1, \frac{1}{H\|u\|}\right)\right) .
$$

Lemma 4.3. Suppose $A_{1}, \cdots, A_{5}$ are absolute positive constants, $f(x)$ and $g(x)$ are algebraic functions in $[a, b]$ and

$$
\begin{aligned}
& \frac{A_{1}}{R} \leq\left|f^{\prime \prime}(x)\right| \leq \frac{A_{2}}{R}, \quad\left|f^{\prime \prime \prime}(x)\right| \leq \frac{A_{3}}{R U}, \quad U \geq 1, \\
& |g(x)| \leq A_{4} G, \quad\left|g^{\prime}(x)\right| \leq A_{5} G U_{1}^{-1}, \quad U_{1} \geq 1
\end{aligned}
$$


$[\alpha, \beta]$ is the image of $[a, b]$ under the mapping $y=f^{\prime}(x)$, then

$$
\begin{aligned}
\sum_{a<n \leq b} g(n) e(f(n))= & e^{\pi i / 4} \sum_{\alpha<u \leq \beta} b_{u} \frac{g\left(n_{u}\right)}{\sqrt{f^{\prime \prime}\left(n_{u}\right)}} e\left(f\left(n_{u}\right)-u n_{u}\right) \\
& +O\left(G \log (\beta-\alpha+2)+G(b-a+R)\left(U^{-1}+U_{1}^{-1}\right)\right) \\
& +O\left(G \min \left[\sqrt{R}, \max \left(\frac{1}{<\alpha>}, \frac{1}{<\beta>}\right)\right]\right),
\end{aligned}
$$

where $n_{u}$ is the solution of $f^{\prime}(n)=u$,

$$
\begin{gathered}
<t>= \begin{cases}\|t\|, & \text { if } t \text { not an integer, } \\
\beta-\alpha, & \text { if } t \text { an integer, }\end{cases} \\
b_{u}= \begin{cases}1, & \text { if } \alpha<u<\beta, \text { or } \alpha, \beta \text { not integers }, \\
1 / 2, & \text { if } \alpha \text { or } \beta \text { are integers, }\end{cases} \\
\sqrt{f^{\prime \prime}}= \begin{cases}\sqrt{f^{\prime \prime}}, & \text { if } f^{\prime \prime}>0, \\
i \sqrt{\left|f^{\prime \prime}\right|}, & \text { if } f^{\prime \prime}<0 .\end{cases}
\end{gathered}
$$

Lemma 4.4. Suppose $f(n)$ is an arithmetic function such that $f(n) \ll n^{\varepsilon}$, $1 \leq v<k$ are fixed integers, $y>1$ is a large parameter, and define

$$
s_{k ; v}(f ; y):=\sum_{\substack{\sqrt{n}^{n_{1}}+\cdots+\sqrt{n_{v}}=\sqrt{n_{v+1}}+\cdots+\sqrt{n_{k}} \\ n_{1}, \cdots, n_{k} \leq y}} \frac{f\left(n_{1}\right) \cdots f\left(n_{k}\right)}{\left(n_{1} \cdots n_{k}\right)^{3 / 4}}, 1 \leq v<k .
$$

Then

$$
\left|s_{k ; v}(f)-s_{k ; v}(f ; y)\right| \ll y^{-1 / 2+\varepsilon}, 1 \leq v<k .
$$

Lemma 4.5. Suppose $A, B \in \mathbb{R}, A \neq 0$, then

$$
\int_{T}^{2 T} \cos (A \sqrt{t}+B) d t \ll T^{1 / 2}|A|^{-1}
$$

Lemma 4.6. Let $\mathcal{S}$ be an inner-product vector space over $\mathbb{C},(a, b)$ denote the inner product in $\mathcal{S}$ and $\|a\|_{0}=\sqrt{(a, a)}$. Suppose that $\xi, \varphi_{1}, \cdots, \varphi_{R}$ are arbitrary vectors in $\mathcal{S}$. Then

$$
\sum_{l \leq R}\left|\left(\xi, \varphi_{l}\right)\right|^{2} \leq\|\xi\|_{0}^{2} \max _{l_{1} \leq R} \sum_{l_{2} \leq R}\left|\left(\varphi_{l_{1}}, \varphi_{l_{2}}\right)\right| .
$$




\section{Proof of Theorem 1}

In order to prove Theorem 1 , we shall prove a large value estimate of $R(x)$. For simplicity and convenience, we consider the function $R_{*}(x):=R(2 \pi x)$.

\section{$5.1 \quad$ Large value estimate of $R_{*}(x)$}

In this subsection, we shall prove the following

Theorem 5.1. Suppose $T \leq x_{1}<x_{2}<\cdots<x_{M} \leq 2 T$ satisfies $\left|R_{*}\left(x_{s}\right)\right| \gg$ $V T^{l-1 / 2}(s=1,2, \cdots, M)$ and $\left|x_{j}-x_{i}\right| \geq V \gg T^{7 / 32} \mathcal{L}^{4}(i \neq j)$, then we have

$$
M \ll T V^{-3} \mathcal{L}^{9}+T^{15 / 4} V^{-12} \mathcal{L}^{41}
$$

Proof. Suppose $V<T_{0}$ is a parameter to be determined later. Let $I$ be any subinterval of $[T, 2 T]$ of length not exceeding $T_{0}$ and let $G=I \cap\left\{x_{1}, x_{2}, \cdots, x_{M}\right\}$. Without loss of generality, suppose $G=\left\{x_{1}, x_{2}, \cdots, x_{M_{0}}\right\}$.

Now let $J=\left[\frac{\mathcal{L} / 2+\log \mathcal{L}-\log V}{2 \log 2}\right]$, then by Lema 3.1 we have

$$
\begin{aligned}
R_{*}(x)=-\frac{4}{2^{l}(l-1) !} \sum_{j=0}^{J} \sum_{m \sim \frac{x^{1 / 2}}{2^{j+1}}} m\left(x-m^{2}\right)^{l-1} \psi\left(\frac{x}{2 m}-\frac{m}{2}+\frac{1}{2}\right)+O\left(\frac{V T^{l-1 / 2}}{\mathcal{L}}\right) \\
=-\frac{4}{2^{l}(l-1) !} \sum_{j_{1}=0}^{l-1}(-1)^{j_{1}}\left(\begin{array}{c}
l-1 \\
j_{1}
\end{array}\right) \sum_{j=0}^{J} \sum_{m \sim \frac{x^{1 / 2}}{2^{j+1}}} x^{l-1-j_{1}} m^{2 j_{1}+1} \psi\left(\frac{x}{2 m}-\frac{m}{2}+\frac{1}{2}\right) \\
+O\left(\frac{V T^{l-1 / 2}}{\mathcal{L}}\right) .
\end{aligned}
$$

By Cauchy's inequality we get

$$
R_{*}^{2}(x) \ll \mathcal{L} \sum_{j_{1}=0}^{l-1} \sum_{j=0}^{J}\left|\sum_{m \sim \frac{x^{1 / 2}}{2^{j+1}}} x^{l-1-j_{1}} m^{2 j_{1}+1} \psi\left(\frac{x}{2 m}-\frac{m}{2}+\frac{1}{2}\right)\right|^{2}+\frac{V^{2} T^{2 l-1}}{\mathcal{L}^{2}} .
$$


Summing over the set $G$ we get

$$
\begin{gathered}
M_{0} V^{2} T^{2 l-1} \ll \sum_{s \leq M_{0}}\left|R_{*}\left(x_{s}\right)\right|^{2} \\
\ll \mathcal{L} \sum_{j_{1}=0}^{l-1} \sum_{j=0}^{J} \sum_{s \leq M_{0}}\left|\sum_{\substack{1 / 2 \\
m \sim \frac{x_{s}}{2^{j+1}}}} x^{l-1-j_{1}} m^{2 j_{1}+1} \psi\left(\frac{x_{s}}{2 m}-\frac{m}{2}+\frac{1}{2}\right)\right|^{2} \\
\ll \mathcal{L}^{2} \sum_{s \leq M_{0}}\left|\sum_{\substack{\sum_{\frac{x_{s} / 2}{2^{j}+1}} \\
2^{l-1-j_{1}}}} m^{2 j_{1}+1} \psi\left(\frac{x_{s}}{2 m}-\frac{m}{2}+\frac{1}{2}\right)\right|^{2}
\end{gathered}
$$

for some fixed pair $\left(j_{1}, j\right)$ with $0 \leq j_{1} \leq l-1,0 \leq j \leq J$. For this fixed pair $\left(j_{1}, j\right)$, let $N=T^{1 / 2} 2^{-j}$ and $H=N^{2 j_{1}+2} V^{-1} T^{-1 / 2-j_{1}} \mathcal{L}^{2}$. By Lemma 4.1 we get

$$
M_{0} V^{2} T^{2 l-1} \ll \mathcal{L}^{2} \sum_{s \leq M_{0}}\left|\sum_{1 \leq h \leq H} c(h) \sum_{\substack{x_{s}^{1 / 2} \\ m \sim \frac{x_{s}}{2^{j}+1}}} x_{s}^{l-1-j_{1}} m^{2 j_{1}+1} e\left(-h\left(\frac{x_{s}}{2 m}-\frac{h m}{2}\right)\right)\right|^{2},
$$

where $c(h)$ is some function such that $c(h) \ll 1 / h$.

For any integer $h>0$, define

$$
S\left(x ; h, j_{1}, j\right):=\sum_{m \sim \frac{x^{1} / 2}{2^{j+1}}} x^{l-1-j_{1}} m^{2 j_{1}+1} e\left(-h\left(\frac{x}{2 m}-\frac{h m}{2}\right)\right) .
$$

Take

$$
f(m)=-h\left(\frac{x}{2 m}-\frac{h m}{2}\right), \quad g(m)=x^{l-1-j_{1}} m^{2 j_{1}+1}
$$

in Lemma 4.3 we get

$$
\begin{aligned}
& =\frac{e^{\pi i / 4}}{i} \sum_{\substack{S\left(x ; h ; j_{1}, j\right) \\
h\left(2^{2 j-1}+\frac{1}{2}\right)<r \leq h\left(2^{2 j+1}+\frac{1}{2}\right) \\
+O\left(T^{l-\frac{1}{2}} \mathcal{L}\right) .}} \frac{b_{r} x^{l-\frac{1}{4}} h^{j_{1}+\frac{3}{4}}}{(2 r-h)^{j_{1}+\frac{5}{4}}} e(-\sqrt{x h(2 r-h)}) \\
& \\
& \quad
\end{aligned}
$$


Insert (5.4) into (5.3) we get

$$
\begin{aligned}
M_{0} V^{2} T^{2 l-1} & \ll \mathcal{L}^{2} \sum_{s \leq M_{0}}\left|D\left(x_{s} ; H, j_{1}, j\right)\right|^{2}+M_{0} T^{2 l-1} \mathcal{L}^{4} \\
& \ll \mathcal{L}^{2} \sum_{s \leq M_{0}}\left|D\left(x_{s} ; H, j_{1}, j\right)\right|^{2},
\end{aligned}
$$

where

$$
D\left(x ; H, j_{1}, j\right)=\sum_{1 \leq h \leq H} c(h) \sum_{h\left(2^{2 j-1}+\frac{1}{2}\right)<r \leq h\left(2^{2 j+1}+\frac{1}{2}\right)} b_{r} \frac{x^{l-1 / 4} h^{j_{1}+3 / 4}}{(2 r-h)^{j_{1}+5 / 4}} e(-\sqrt{x h(2 r-h)}) .
$$

Let

$$
\gamma\left(n ; H, j_{1}, j\right)=\sum_{\substack{n=h(2 r-h), 1 \leq h \leq H \\ h\left(2^{2 j-1}+\frac{1}{2}\right)<r \leq h\left(2^{2 j+1}+\frac{1}{2}\right)}} \frac{b_{r} c(h) h^{j_{1}+3 / 2}}{(2 r-h)^{j_{1}+1 / 2}}
$$

and let $N_{0}=H^{2}\left(2^{2 j+1}+1 / 2\right)$. Then it is easy to see that $\gamma\left(n ; H, j_{1}, j\right) \ll d(n)$ and $N_{0} \ll T V^{-2} \mathcal{L}^{4}$. Thus we have

$$
\begin{aligned}
M_{0} V^{2} & \ll \mathcal{L}^{2} T^{1 / 2} \sum_{s \leq M_{0}}\left|\sum_{n \leq N_{0}} \frac{\gamma\left(n ; H, j_{1}, j\right)}{n^{3 / 4}} e\left(-\sqrt{n x_{s}}\right)\right|^{2} \\
& \ll \mathcal{L}^{2} T^{1 / 2} \sum_{s \leq M_{0}}\left|\sum_{v} \sum_{n \sim N_{0} 2^{-v-1}} \frac{\gamma\left(n ; H, j_{1}, j\right)}{n^{3 / 4}} e\left(-\sqrt{n x_{s}}\right)\right|^{2} \\
& \ll \mathcal{L}^{3} T^{1 / 2} \sum_{v} \sum_{s \leq M_{0}}\left|\sum_{n \sim N_{0} 2^{-v-1}} \frac{\gamma\left(n ; H, j_{1}, j\right)}{n^{3 / 4}} e\left(-\sqrt{n x_{s}}\right)\right|^{2} \\
& \ll \mathcal{L}^{4} T^{1 / 2} \sum_{s \leq M_{0}}\left|\sum_{n \sim N_{0} 2^{-v-1}} \frac{\gamma\left(n ; H, j_{1}, j\right)}{n^{3 / 4}} e\left(-\sqrt{n x_{s}}\right)\right|^{2}
\end{aligned}
$$

for some $0 \leq v \ll \mathcal{L}$, where in the third "«" we used the Cauchy's inequality again. Let $N_{1}=N_{0} 2^{-v-1}$, then $N_{1} \ll T V^{-2} \mathcal{L}^{4}$.

The procedure below is the same as the approach of the proof of Theorem $13.8 \mathrm{in}$ Ivić[15], so we give only an outline. Take $\xi=\left\{\xi_{n}\right\}_{n=1}^{\infty}$ with $\xi_{n}=\gamma\left(n ; H, j_{1}, j\right) n^{-3 / 4}$ for $n \sim N_{1}$ and zero otherwise, and take $\varphi_{s}=\left\{\varphi_{s, n}\right\}_{n=1}^{\infty}$ with $\varphi_{s, n}=e\left(-\sqrt{n x_{s}}\right)$ for 
$n \sim N_{1}$ and zero otherwise. Then

$$
\begin{aligned}
& \left(\xi, \varphi_{s}\right)=\sum_{n \sim N_{1}} \frac{\gamma\left(n ; H, j_{1}, j\right)}{n^{3 / 4}} e\left(-\sqrt{n x_{s}}\right), \\
& \left(\varphi_{l_{1}}, \varphi_{l_{2}}\right)=\sum_{n \sim N_{1}} e\left(\sqrt{n}\left(\sqrt{x_{s_{1}}}-\sqrt{x_{s_{2}}}\right)\right), \\
& \|\xi\|_{0}^{2}=\sum_{n \sim N_{1}} \frac{\left|\gamma\left(n ; H, j_{1}, j\right)\right|^{2}}{n^{3 / 2}} \ll N_{1}^{-3 / 2} \sum_{n \sim N_{1}} d^{2}(n) \ll N_{1}^{-1 / 2} \mathcal{L}^{3} .
\end{aligned}
$$

By Lemma 4.6 we get

$$
\begin{aligned}
(5.7) M_{0} V^{2} & \ll \mathcal{L}^{7} T^{1 / 2} N_{1}^{-1 / 2} \max _{s_{1} \leq M_{0}} \sum_{s_{2} \leq M_{0}}\left|\sum_{n \sim N_{1}} e\left(\sqrt{n}\left(\sqrt{x_{s_{1}}}-\sqrt{x_{s_{2}}}\right)\right)\right| \\
& \ll \mathcal{L}^{7} T^{1 / 2} N_{1}^{1 / 2}+\mathcal{L}^{7} T^{1 / 2} N_{1}^{-1 / 2} \max _{s_{1} \leq M_{0}} \sum_{s_{2} \leq M_{0}, s_{2} \neq s_{1}}\left|\sum_{n \sim N_{1}} e\left(\sqrt{n}\left(\sqrt{x_{s_{1}}}-\sqrt{x_{s_{2}}}\right)\right)\right| .
\end{aligned}
$$

By the Kuzmin-Landau inequality and the exponent pair $(4 / 18,11 / 18)$ we get

$$
\begin{aligned}
\sum_{n \sim N_{1}} e\left(\sqrt{n}\left(\sqrt{x_{s_{1}}}-\sqrt{x_{s_{2}}}\right)\right) & \ll \frac{\sqrt{N_{1}}}{\left|\sqrt{x_{s_{1}}}-\sqrt{x_{s_{2}}}\right|}+\left(\frac{\left|\sqrt{x_{s_{1}}}-\sqrt{x_{s_{2}}}\right|}{\sqrt{N_{1}}}\right)^{\frac{4}{18}} N_{1}^{\frac{11}{18}} \\
& \ll \frac{\sqrt{N_{1} T}}{\left|x_{s_{1}}-x_{s_{2}}\right|}+\left(\frac{\left|x_{s_{1}}-x_{s_{2}}\right|}{\sqrt{N_{1} T}}\right)^{\frac{4}{18}} N_{1}^{\frac{11}{18}} \\
& \ll \frac{\sqrt{N_{1} T}}{\left|x_{s_{1}}-x_{s_{2}}\right|}+T^{-1 / 9} T_{0}^{2 / 9} N_{1}^{1 / 2},
\end{aligned}
$$

where we used the mean value theorem and the estimate $\left|x_{s_{1}}-x_{s_{2}}\right| \leq T_{0}$.

Insert this estimate into (5.7) we get

$$
\begin{aligned}
M_{0} V^{2} & \ll \mathcal{L}^{7} T^{1 / 2} N_{1}^{1 / 2}+\mathcal{L}^{7} T^{1 / 2} N_{1}^{-1 / 2} \\
& \times \max _{s_{1} \leq M_{0}} \sum_{s_{2} \leq M_{0}, s_{2} \neq s_{1}}\left(\frac{\sqrt{N_{1} T}}{\left|x_{s_{1}}-x_{s_{2}}\right|}+T^{-1 / 9} T_{0}^{2 / 9} N_{1}^{1 / 2}\right) \\
& \ll \mathcal{L}^{7} T^{1 / 2} N_{1}^{1 / 2}+\mathcal{L}^{7} T V^{-1}+\mathcal{L}^{7} M_{0} T^{1 / 2-1 / 9} T_{0}^{2 / 9} \\
& \ll \mathcal{L}^{9} T V^{-1}+\mathcal{L}^{7} M_{0} T^{7 / 18} T_{0}^{2 / 9}
\end{aligned}
$$

where we used the facts that $\left\{x_{s}\right\}$ is $V$-spaced and $N_{1} \ll T V^{-2} \mathcal{L}^{4}$. Take $T_{0}=$ $V^{9} T^{-7 / 4} \mathcal{L}^{-32}$, it is easy to check that $T_{0} \gg V$ if $V \gg T^{7 / 32} \mathcal{L}^{4}$. We get for this $T_{0}$ that

$$
M_{0} \ll \mathcal{L}^{9} T V^{-3}
$$


Now we divide the interval $[T, 2 T]$ into $O\left(1+T / T_{0}\right)$ subintervals of length not exceeding $T_{0}$. In each interval of this type, the number of $x_{s}$ is at most $O\left(\mathcal{L}^{9} T V^{-3}\right)$

. So we have

$$
M \ll \mathcal{L}^{9} T V^{-3}\left(1+\frac{T}{T_{0}}\right) \ll \mathcal{L}^{9} T V^{-3}+\mathcal{L}^{41} T^{15 / 4} V^{-12} .
$$

This completes the proof of Theorem 5.1.

\subsection{Proof of Theorem 1}

Now we prove Theorem 1 . When $A=0$, Theorem 1 is trivial. When $0<A<2$, it follows from (1.11) and the Hölder's inequality. So later we always suppose $A>2$. It suffices for us to prove the estimate

$$
\int_{T}^{2 T}\left|R_{*}(x)\right|^{A} d x \ll \begin{cases}T^{1+\frac{3 A}{4}+A(l-1)} \log ^{41} T, & \text { if } 2<A \leq 262 / 27 \\ T^{\frac{15+3399 A}{416}+A(l-1)} \log ^{4 A+1} T, & \text { if } A>262 / 27\end{cases}
$$

Suppose $x^{\varepsilon}<y \leq x / 2$, by (3.16) we get

$$
\begin{aligned}
&\left|R_{*}(x+y)-R_{*}(x)\right| \\
& \ll \sum_{x<n \leq x+y} f_{R}(n)+\left|(x+y)^{l+1 / 2}-x^{l+1 / 2}\right|+\left|(x+y)^{l}-x^{l}\right| \\
& \ll x^{l-1 / 2} \sum_{x<n \leq x+y} d(n)+x^{l-1 / 2} y \\
& \ll x^{l-1 / 2} y \log x,
\end{aligned}
$$

where we used the well-known estimate

$$
\sum_{x<n \leq x+y} d(n) \ll y \log x
$$

and the obvious bounds $f_{R}(n) \ll n^{l-1 / 2} d(n)$. So there exists an absolute constant $c_{0}$ such that

$$
\left|R_{*}(x+y)-R_{*}(x)\right| \leq c_{0} x^{l-1 / 2} y \log x,
$$

which impies that if $\left|R_{*}(x)\right| \geq 2 c_{0} x^{l-1 / 2} y \log x$, then

$$
\left|R_{*}(x+y)\right| \geq\left|R_{*}(x)\right|-\left|R_{*}(x+y)-R_{*}(x)\right| \geq c_{0} x^{l-1 / 2} y \log x .
$$


From (5.11) and a similar argument to (13.70) of Ivić[15] we may write

$$
\int_{T}^{2 T}\left|R_{*}(x)\right|^{A} d x \ll T^{1+A\left(l-\frac{1}{4}\right)} \mathcal{L}+\sum_{V} V \sum_{r \leq N_{V}}\left|R_{*}\left(x_{r}\right)\right|^{A},
$$

where $T^{1 / 4} \leq V=2^{m} \leq T^{131 / 416} \mathcal{L}^{4}, V T^{l-1 / 2}<\left|R_{*}\left(x_{r}\right)\right| \leq 2 V T^{l-1 / 2}\left(r=1, \cdots, N_{V}\right)$ and $\left|t_{r}-t_{s}\right| \geq V$ for $r \neq s \leq N=N_{V}$.

If $2<A \leq 11$, then by Theorem 5.1 we get (recall $V \ll T^{131 / 416} \mathcal{L}^{4}$ )

$$
\begin{aligned}
& V \sum_{r \leq N_{V}}\left|R_{*}\left(x_{r}\right)\right|^{A} \ll T^{A(l-1 / 2)} N_{V} V^{A+1} \\
& \ll T^{A(l-1 / 2)}\left(\mathcal{L}^{9} T V^{A-2}+\mathcal{L}^{41} T^{\frac{15}{4}} V^{A-11}\right) \\
& \ll T^{A(l-1 / 2)}\left(T^{1+\frac{131}{416}(A-2)} \mathcal{L}^{4 A+1}+T^{1+\frac{A}{4}} \mathcal{L}^{41}\right) \\
& \ll T^{A(l-1 / 2)}\left(T^{\frac{154+131 A}{416}} \mathcal{L}^{4 A+1}+T^{1+\frac{A}{4}} \mathcal{L}^{41}\right) .
\end{aligned}
$$

If $A>11$, then by Theorem 5.1 we get

$$
\begin{aligned}
& V \sum_{r \leq N_{V}}\left|R_{*}\left(x_{r}\right)\right|^{A} \ll T^{A(l-1 / 2)} N_{V} V^{A+1} \\
& \ll T^{A(l-1 / 2)}\left(\mathcal{L}^{9} T V^{A-2}+\mathcal{L}^{41} T^{\frac{15}{4}} V^{A-11}\right) \\
& \ll T^{A(l-1 / 2)}\left(T^{1+\frac{131}{416}(A-2)} \mathcal{L}^{4 A+1}+T^{\frac{15}{4}+\frac{131}{416}(A-11)} \mathcal{L}^{4 A-3}\right) \\
& \ll T^{A(l-1 / 2)+\frac{154+131 A}{416}} \mathcal{L}^{4 A+1} .
\end{aligned}
$$

Now (5.10) follows from (5.12)-(5.14) by noting that $(154+131 A) / 416 \leq 1+A / 4$ for $2<A \leq 262 / 27$ and $(154+131 A) / 416>1+A / 4$ for $A>262 / 27$.

\section{Proof of Theorem 2}

In this section we shall prove Theorem 2. Suppose that $3 \leq k<A_{0}, A_{0}>9$ is a fixed real number such that (2.3) holds. It suffices for us to evaluate the integral $\int_{T}^{2 T} R_{*}^{k}(x) d x$, where $T$ is a large real number.

Suppose $H$ is a large parameter to be determined later. By Lemma 3.1 and 
Lemma 4.2 we have

$$
\begin{aligned}
& R_{*}(x)=F(x)+O\left(T^{l-1 / 2} G(x)\right), \\
& F(x)=\frac{2^{1-l}}{(l-1) ! \pi i} \sum_{1 \leq|h| \leq H} \frac{1}{h} \sum_{m \leq \sqrt{x}} m\left(x-m^{2}\right)^{l-1} e\left(h\left(\frac{x}{2 m}-\frac{m}{2}+\frac{l}{2}\right)\right), \\
& G(x)=\sum_{m \leq \sqrt{2 T}} \min \left(1, \frac{1}{H\left\|\frac{x}{2 m}-\frac{m}{2}+\frac{l}{2}\right\|}\right) .
\end{aligned}
$$

\subsection{The upper bound of $\int_{T}^{2 T} G(x) d x$}

In this subsection we shall prove the following Lemma 6.1.

Lemma 6.1. We have

$$
\int_{T}^{2 T} G(x) d x \ll T^{3 / 2} H^{-1} \mathcal{L} .
$$

Proof. Obviously we have

$$
\begin{aligned}
G(x) \ll & \sum_{2 m \leq \sqrt{2 T}} \min \left(1, \frac{1}{H\left\|\frac{x}{4 m}+\frac{1}{2}\right\|}\right) \\
& +\sum_{\substack{2 m-1 \leq \sqrt{2 T}\\
}} \min \left(1, \frac{1}{H\left\|\frac{x}{2(2 m-1)}\right\|}\right) \\
& \ll G_{1}(x)+G_{2}(x),
\end{aligned}
$$

where

$$
\begin{aligned}
G_{1}(x) & =\sum_{m \leq 4 \sqrt{T}} \min \left(1, \frac{1}{H\left\|\frac{x}{m}+\frac{1}{2}\right\|}\right), \\
G_{2}(x) & =\sum_{m \leq 4 \sqrt{T}} \min \left(1, \frac{1}{H\left\|\frac{x}{m}\right\|}\right) .
\end{aligned}
$$

Thus

$$
\int_{T}^{2 T} G(x) d x \ll \int_{T}^{2 T} G_{1}(x) d x+\int_{T}^{2 T} G_{2}(x) d x .
$$


We have

$$
\begin{aligned}
\int_{T}^{2 T} G_{1}(x) d x & \ll \sum_{m \leq 4 \sqrt{T}} \int_{T}^{2 T} \min \left(1, \frac{1}{H\left\|\frac{x}{m}+\frac{1}{2}\right\|}\right) d x \\
& \ll \sum_{m \leq 4 \sqrt{T}} m \int_{\frac{T}{m}}^{\frac{2 T}{m}} \min \left(1, \frac{1}{H\left\|x+\frac{1}{2}\right\|}\right) d x \\
& \ll T \sum_{m \leq 4 \sqrt{T}} \int_{0}^{1 / 2}\left(1, \frac{1}{H\left\|x+\frac{1}{2}\right\|}\right) d x \\
& \ll T^{3 / 2} \int_{0}^{1 / 2}\left(1, \frac{1}{H\left\|x+\frac{1}{2}\right\|}\right) d x \\
& \ll T^{3 / 2} H^{-1} \mathcal{L} .
\end{aligned}
$$

Similarly we have

$$
\int_{T}^{2 T} G_{2}(x) d x \ll T^{3 / 2} H^{-1} \mathcal{L} .
$$

Now Lemma 6.1 follows from (6.3)-(6.6).

\subsection{An analogue of Voronoi's formula for $R_{*}(x)$}

In this subsection we shall give an analogue of Voronoi's formula for $R_{*}(x)$. Our main tool is Lemma 4.2 and Lemma 4.3.

We begin with $F(x)$. From the definition of $F(x)$ we have

$$
\begin{aligned}
(6.7) F(x)= & \frac{2^{1-l}}{(l-1) ! \pi i} \sum_{1 \leq|h| \leq H} \frac{1}{h} \sum_{m \leq \sqrt{x}} m\left(x-m^{2}\right)^{l-1} e\left(h\left(\frac{x}{2 m}-\frac{m}{2}-\frac{l}{2}\right)\right) \\
= & \frac{2^{1-l}}{(l-1) ! \pi i} \sum_{j_{1}=0}^{l-1}(-1)^{j_{1}}\left(\begin{array}{c}
l-1 \\
j_{1}
\end{array}\right) \sum_{1 \leq|h| \leq H} \frac{1}{h} \sum_{m \leq \sqrt{x}} x^{l-1-j_{1}} m^{2 j_{1}+1} \\
& \times e\left(h\left(\frac{x}{2 m}-\frac{m}{2}-\frac{l}{2}\right)\right) \\
= & \frac{2^{1-l}}{(l-1) !} \sum_{j_{1}=0}^{l-1}(-1)^{j_{1}}\left(\begin{array}{c}
l-1 \\
j_{1}
\end{array}\right) F\left(x ; j_{1}\right),
\end{aligned}
$$


say, where

$$
F\left(x ; j_{1}\right):=\frac{1}{\pi i} \sum_{1 \leq|h| \leq H} \frac{1}{h} \sum_{m \leq \sqrt{x}} x^{l-1-j_{1}} m^{2 j_{1}+1} e\left(h\left(\frac{x}{2 m}-\frac{m}{2}-\frac{l}{2}\right)\right) .
$$

Let $J=[(\mathcal{L}-\log \mathcal{L}) / 2 \log 2]$ we get

$$
\begin{aligned}
= & \frac{1}{\pi i} \sum_{-H \leq h \leq-1} \frac{e(-l h / 2)}{h} \sum_{j=0}^{J} \sum_{m \sim \sqrt{x} 2^{-j-1}} x^{l-1-j_{1}} m^{2 j_{1}+1} e\left(h\left(\frac{x}{2 m}-\frac{m}{2}\right)\right) \\
& +\frac{1}{\pi i} \sum_{1 \leq h \leq H} \frac{e(-l h / 2)}{h} \sum_{j=0}^{J} \sum_{m \sim \sqrt{x} 2^{-j-1}} x^{l-1-j_{1}} m^{2 j_{1}+1} e\left(h\left(\frac{x}{2 m}-\frac{m}{2}\right)\right)+O\left(x^{l-1} \mathcal{L}^{2}\right) \\
= & -\frac{1}{\pi i} \sum_{1 \leq h \leq H} \frac{e(l h / 2)}{h} \sum_{j=0}^{J} \sum_{m \sim \sqrt{x} 2^{-j-1}} x^{l-1-j_{1}} m^{2 j_{1}+1} e\left(-h\left(\frac{x}{2 m}-\frac{m}{2}\right)\right) \\
& +\frac{1}{\pi i} \sum_{1 \leq h \leq H} \frac{e(-l h / 2)}{h} \sum_{j=0}^{J} \sum_{m \sim \sqrt{x} 2^{-j-1}} x^{l-1-j_{1}} m^{2 j_{1}+1} e\left(h\left(\frac{x}{2 m}-\frac{m}{2}\right)\right)+O\left(x^{l-1} \mathcal{L}^{2}\right) \\
= & -\frac{\Sigma_{3}}{\pi i}+\frac{\Sigma_{3}}{\pi i}+O\left(x^{l-1} \mathcal{L}^{2}\right),
\end{aligned}
$$

where

$$
\Sigma_{3}=\sum_{1 \leq h \leq H} \frac{e(l h / 2)}{h} \sum_{j=0}^{J} S\left(x ; h, j_{1}, j\right)
$$

with $S\left(x ; h, j_{1}, j\right)$ defined in Section 5.1.

Insert the formula (5.4) into $\Sigma_{3}$ we get

$$
\begin{aligned}
& \Sigma_{3}=e^{-\pi i / 4} \sum_{1 \leq h \leq H} \frac{e(l h / 2)}{h} \sum_{j=0}^{J} \sum_{h\left(2^{2 j-1}+\frac{1}{2}\right)<r \leq h\left(2^{2 j+1}+\frac{1}{2}\right)} \\
& \quad \frac{b_{r} x^{l-1 / 4} h^{j_{1}+3 / 4}}{(2 r-h)^{j_{1}+5 / 4}} e(-\sqrt{x h(2 r-h)})+O\left(T^{l-1 / 2} \mathcal{L}^{3}\right) \\
& =e^{-\pi i / 4} \sum_{1 \leq h \leq H} \frac{e(l h / 2)}{h} \sum_{h<r \leq h\left(2^{2 J+1}+1 / 2\right)}^{\prime} \frac{x^{l-1 / 4} h^{j_{1}+3 / 4}}{(2 r-h)^{j_{1}+5 / 4}} e(-\sqrt{x h(2 r-h)}) \\
& +O\left(T^{l-1 / 2} \mathcal{L}^{3}\right),
\end{aligned}
$$


where in the last sum the symbol " " means that if $h$ is an even integer, then the term $r=h\left(2^{2 J+1}+1 / 2\right)$ should be halved .

Inserting (6.9) into (6.8) we get

$$
\begin{aligned}
F\left(x ; j_{1}\right) & =\frac{1}{\pi i} \sum_{1 \leq h \leq H} \frac{e(l h / 2)}{h} \sum_{h<r \leq h\left(2^{2 J+1}+1 / 2\right)}^{\prime} \frac{x^{l-1 / 4} h^{j_{1}+3 / 4}}{(2 r-h)^{j_{1}+5 / 4}} \\
& \times\left(e\left(\sqrt{x h(2 r-h)}+\frac{1}{8}\right)-e\left(\sqrt{x h(2 r-h)}-\frac{1}{8}\right)\right)+O\left(x^{l-1 / 2} \mathcal{L}^{3}\right) \\
=\frac{2 x^{l-1 / 4}}{\pi} & \sum_{1 \leq h \leq H} \frac{e(l h / 2)}{h} \sum_{h<r \leq h\left(2^{2 J+1}+1 / 2\right)}^{\prime} \frac{h^{j_{1}+3 / 4}}{(2 r-h)^{j_{1}+5 / 4}} \sin \left(2 \pi \sqrt{x h(2 r-h)}+\frac{\pi}{4}\right) \\
=\frac{2 x^{l-1 / 4}}{\pi} & \sum_{1 \leq h \leq H} \frac{e(l h / 2)}{h} \sum_{h<r \leq h\left(2^{2 J+1}+1 / 2\right)}^{\prime} \frac{h^{j_{1}+3 / 4}}{(2 r-h)^{j_{1}+5 / 4}} \cos \left(2 \pi \sqrt{x h(2 r-h)}-\frac{\pi}{4}\right) \\
& +O\left(x^{l-1 / 2} \mathcal{L}^{3}\right) .
\end{aligned}
$$

From (6.7) and (6.10) we get

$$
\begin{gathered}
F(x)=\frac{2^{2-l} x^{l-1 / 4}}{(l-1) ! \pi} \sum_{1 \leq h \leq H} \sum_{h<r \leq h\left(2^{2 J+1}+1 / 2\right)}^{\prime} \cos \left(2 \pi \sqrt{x h(2 r-h)}-\frac{\pi}{4}\right) \\
\times \sum_{j_{1}=0}^{l-1}(-1)^{j_{1}}\left(\begin{array}{c}
l-1 \\
j_{1}
\end{array}\right) \frac{e(l h / 2) h^{j_{1}-1 / 4}}{(2 r-h)^{j_{1}+5 / 4}}+O\left(x^{l-1 / 2} \mathcal{L}^{3}\right) \\
=\frac{2^{2-l} x^{l-1 / 4}}{(l-1) ! \pi} \sum_{1 \leq h \leq H} \sum_{h<r \leq h\left(2^{2 J+1}+1 / 2\right)}^{\prime} \frac{e(l h / 2)}{h^{1 / 4}(2 r-h)^{5 / 4}}\left(1-\frac{h}{2 r-h}\right)^{l-1} \\
\times \cos \left(2 \pi \sqrt{x h(2 r-h)}-\frac{\pi}{4}\right)+O\left(x^{l-1 / 2} \mathcal{L}^{3}\right)
\end{gathered}
$$

Define

$$
\tau_{l}(n ; H, T):=\sum_{\substack{n=h(2 r-h), 1 \leq h \leq H \\ h<r \leq h\left(2^{2 J+1}+1 / 2\right)}} \frac{e(l h / 2) h^{1 / 2}}{(2 r-h)^{1 / 2}}\left(1-\frac{h}{2 r-h}\right)^{l-1} .
$$

We then have

$$
F(x)=\frac{2^{2-l} x^{l-1 / 4}}{(l-1) ! \pi} \sum_{1 \leq n \leq H^{2}\left(2^{2 J+1}+1 / 2\right)} \frac{\tau_{l}(n ; H, T)}{n^{3 / 4}} \cos \left(2 \pi \sqrt{x n}-\frac{\pi}{4}\right)+O\left(x^{l-1 / 2} \mathcal{L}^{3}\right),
$$


where the " " terms for which $2 \mid h$ and $r=h\left(2^{2 J+1}+1 / 2\right)$ are absorbed into the error term.

Obviously that $\left|\tau_{l}(n ; H, T)\right| \leq d(n)$. By the definition of $J$ we see that if $n \leq$ $\min \left(H, T \mathcal{L}^{-2}\right)$, then $\tau_{l}(n ; H, T)=\tau_{l}(n)$, where $\tau_{l}(n)$ was defined in Section 2.1.

From (6.1) and (6.11) we get the following Proposition, which is an analogue of the Voronoi's formula.

Proposition 6.1. Suppose $T \leq x \leq 2 T, H \geq T, J=[(\mathcal{L}-\log \mathcal{L}) / 2 \log 2]$. Then we have

$$
\begin{gathered}
R_{*}(x)=\frac{2^{2-l} x^{l-1 / 4}}{(l-1) ! \pi} \sum_{\substack{1 \leq n \leq H^{2}\left(2^{2 J+1}+1 / 2\right) \\
n^{3 / 4}}} \frac{\tau(n ; H, T)}{{ }^{l-1}} \cos \left(2 \pi \sqrt{x n}-\frac{\pi}{4}\right) \\
+O\left(T^{l-1 / 2} G(x)+T^{l-1 / 2} \mathcal{L}^{3}\right),
\end{gathered}
$$

where $\tau_{l}(n ; H, T)=\tau_{l}(n)$ for $n \leq T \mathcal{L}^{-2}$.

Now suppose $T^{\varepsilon}<y \leq T \mathcal{L}^{-2}$ is a parameter to be determined. Define

$$
\begin{aligned}
& F_{1}(x):=\theta_{l} x^{l-1 / 4} \sum_{1 \leq n \leq y} \frac{\tau_{l}(n)}{n^{3 / 4}} \cos \left(2 \pi \sqrt{x n}-\frac{\pi}{4}\right), \\
& F_{2}(x):=F(x)-F_{1}(x)
\end{aligned}
$$

where $\theta_{l}:=\frac{2^{2-l}}{(l-1) ! \pi}$.

\subsection{Evaluation of the integral $\int_{T}^{2 T} F_{1}^{k}(x) d x$}

The $k$-th power moment of $F_{1}(x)$ provides the main term in Theorem 2 , so in this subsection we shall evaluate the integral $\int_{T}^{2 T} F_{1}^{k}(x) d x$. For simplicity we set $\mathbb{I}=\{0,1\}$ and

$$
\mathbb{N}^{k}=\left\{\mathbf{n}: \mathbf{n}=\left(n_{1}, \cdots, n_{k}\right), n_{j} \in \mathbb{N}, 1 \leq j \leq k\right\}
$$

For each element $\mathbf{i}=\left(i_{1}, \cdots, i_{k-1}\right) \in \mathbb{I}^{k-1}$, put $|\mathbf{i}|=i_{1}+\cdots+i_{k-1}$. By the elementary formula

$$
\cos a_{1} \cdots \cos a_{k}=\frac{1}{2^{k-1}} \sum_{\mathbf{i} \in \mathbb{I}^{k-1}} \cos \left(a_{1}+(-1)^{i_{1}} a_{2}+(-1)^{i_{2}} a_{3}+\cdots+(-1)^{i_{k-1}} a_{k}\right)
$$


we have

$$
\begin{aligned}
F_{1}^{k}(x) & =\theta_{l}^{k} x^{k\left(l-\frac{1}{4}\right)} \sum_{n_{1} \leq y} \cdots \sum_{n_{k} \leq y} \frac{\tau_{l}\left(n_{1}\right) \cdots \tau_{l}\left(n_{k}\right)}{\left(n_{1} \cdots n_{k}\right)^{3 / 4}} \prod_{j=1}^{k} \cos \left(2 \pi \sqrt{n_{j} x}-\frac{\pi}{4}\right) \\
& =\frac{\theta_{l}^{k} x^{k(l-1 / 4)}}{2^{k-1}} \sum_{\mathbf{i} \in \mathbb{I}^{k-1}} \sum_{n_{1} \leq y} \cdots \sum_{n_{k} \leq y} \frac{\tau_{l}\left(n_{1}\right) \cdots \tau_{l}\left(n_{k}\right)}{\left(n_{1} \cdots n_{k}\right)^{3 / 4}} \cos \left(2 \pi \sqrt{x} \alpha(\mathbf{n} ; \mathbf{i})-\frac{\pi}{4} \beta(\mathbf{i})\right),
\end{aligned}
$$

where

$$
\begin{aligned}
\alpha(\mathbf{n} ; \mathbf{i}): & =\sqrt{n_{1}}+(-1)^{i_{1}} \sqrt{n_{2}}+(-1)^{i_{2}} \sqrt{n_{3}}+\cdots+(-1)^{i_{k-1}} \sqrt{n_{k}}, \\
\beta(\mathbf{i}): & =1+(-1)^{i_{1}}+(-1)^{i_{2}}+\cdots+(-1)^{i_{k-1}} .
\end{aligned}
$$

Thus we can write

$$
F_{1}^{k}(x)=\frac{\theta_{l}^{k}}{2^{k-1}}\left(S_{1}(x)+S_{2}(x)\right)
$$

where

$$
\begin{aligned}
& S_{1}(x): \quad=x^{k(l-1 / 4)} \sum_{\mathbf{i} \in \mathbb{I}^{k-1}} \cos \left(-\frac{\pi \beta(\mathbf{i})}{4}\right) \sum_{\substack{n_{j} \leq y, 1 \leq j \leq k \\
\alpha(\mathbf{n} ; \mathbf{i})=0}} \frac{\tau_{l}\left(n_{1}\right) \cdots \tau_{l}\left(n_{k}\right)}{\left(n_{1} \cdots n_{k}\right)^{3 / 4}},
\end{aligned}
$$

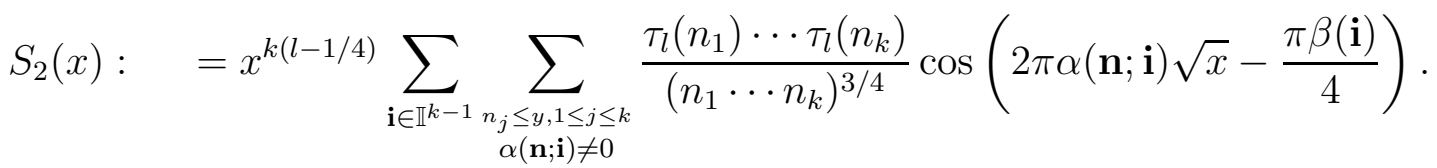

First consider the contribution of $S_{1}(x)$. We have

$$
\int_{T}^{2 T} S_{1}(x) d x=\sum_{\mathbf{i} \in \mathbb{I}^{k-1}} \cos \left(-\frac{\pi \beta(\mathbf{i})}{4}\right) \sum_{\substack{n_{j} \leq y, 1 \leq j \leq k \\ \alpha(\mathbf{n} ; \mathbf{i})=0}} \frac{\tau_{l}\left(n_{1}\right) \cdots \tau_{l}\left(n_{k}\right)}{\left(n_{1} \cdots n_{k}\right)^{3 / 4}} \int_{T}^{2 T} x^{k(l-1 / 4)} d x .
$$

It is easily seen that if $\alpha(\mathbf{n} ; \mathbf{i})=0$, then $1 \leq|\mathbf{i}| \leq k-1$. So

$$
\sum_{\substack{n_{j} \leq y, 1 \leq j \leq k \\ \alpha(\mathbf{n} ; \mathbf{i})=0}} \frac{\tau_{l}\left(n_{1}\right) \cdots \tau_{l}\left(n_{k}\right)}{\left(n_{1} \cdots n_{k}\right)^{3 / 4}}=s_{k ;|\mathbf{i}|}\left(\tau_{l} ; y\right)
$$

By Lemma 4.4 we get

$$
\int_{T}^{2 T} S_{1}(x) d x=B_{k}^{*}\left(\tau_{l}\right) \int_{T}^{2 T} x^{k(l-1 / 4)} d x+O\left(T^{1+k(l-1 / 4)+\varepsilon} y^{-1 / 2}\right),
$$


where

$$
B_{k}^{*}\left(\tau_{l}\right):=\sum_{\mathbf{i} \in \mathbb{I}^{k-1}} \cos \left(-\frac{\pi \beta(\mathbf{i})}{4}\right) \sum_{\substack{\mathbf{n} \in \mathbb{N}^{k} \\ \alpha(\mathbf{n} ; \mathbf{i})=0}} \frac{\tau_{l}\left(n_{1}\right) \cdots \tau_{l}\left(n_{k}\right)}{\left(n_{1} \cdots n_{k}\right)^{3 / 4}} .
$$

For any $\mathbf{i} \in \mathbb{I}^{k-1} \backslash \mathbf{0}$, let

$$
S\left(\tau_{l} ; \mathbf{i}\right):=\sum_{\substack{\mathbf{n} \in \mathbb{N}^{k} \\ \alpha(\mathbf{n} ; \mathbf{i})=0}} \frac{\tau_{l}\left(n_{1}\right) \cdots \tau\left(n_{k}\right)}{\left(n_{1} \cdots n_{k}\right)^{3 / 4}} .
$$

It is easily seen that if $|\mathbf{i}|=\left|\mathbf{i}^{\prime}\right|$ or $|\mathbf{i}|+\left|\mathbf{i}^{\prime}\right|=k$, then

$$
S\left(\tau_{l} ; \mathbf{i}\right)=S\left(\tau_{l} ; \mathbf{i}^{\prime}\right)=s_{k ; \mathbf{i} \mid}\left(\tau_{l}\right) .
$$

From $(-1)^{j}=1-2 j(j=0,1)$ we also have $\beta(\mathbf{i})=k-2|\mathbf{i}|$. So we get

$$
\begin{aligned}
B_{k}^{*}\left(\tau_{l}\right) & =\sum_{v=1}^{k-1} \sum_{|\mathbf{i}|=v} \cos \left(-\frac{\pi \beta(\mathbf{i})}{4}\right) S\left(\tau_{l} ; \mathbf{i}\right) \\
& =\sum_{v=1}^{k-1} s_{k ; v}\left(\tau_{l}\right) \cos \frac{\pi(k-2 v)}{4} \sum_{|\mathbf{i}|=v} 1 \\
& =\sum_{v=1}^{k-1}\left(\begin{array}{c}
k-1 \\
v
\end{array}\right) s_{k ; v}\left(\tau_{l}\right) \cos \frac{\pi(k-2 v)}{4}=B_{k}\left(\tau_{l}\right) .
\end{aligned}
$$

Now we consider the contribution of $S_{2}(x)$. By Lemma 4.5 we get

$$
\begin{aligned}
& \int_{T}^{2 T} S_{2}(x) d x \ll T^{1 / 2+k(l-1 / 4)} U_{k}(y), \\
& U_{k}(y):=\sum_{\mathbf{i} \in \mathbb{I}^{k-1}} \sum_{\substack{n_{j} \leq y, 1 \leq j \leq k \\
\alpha(\mathbf{n} ; \mathbf{i} \neq \neq 0}} \frac{d\left(n_{1}\right) \cdots d\left(n_{k}\right)}{\left(n_{1} \cdots n_{k}\right)^{3 / 4}|\alpha(\mathbf{n} ; \mathbf{i})|} .
\end{aligned}
$$

In [30] the author proved

$$
U_{k}(y) \ll y^{s(k)+\varepsilon},
$$

where $s(k)$ was defined in Section 2.1. Thus

$$
\int_{T}^{2 T} S_{2}(x) d x \ll T^{1 / 2+k(l-1 / 4)+\varepsilon} y^{s(k)} .
$$


Hence from (6.15)-(6.22) we get

Lemma 6.2. For fixed $k \geq 3$, we have

$$
\begin{aligned}
\int_{T}^{2 T} F_{1}^{k}(x) d x= & \frac{2^{1+k-l k} l^{k} B_{k}\left(\tau_{l}\right)}{(l !)^{k} \pi^{k}} \int_{T}^{2 T} x^{k\left(l-\frac{1}{4}\right)} d x \\
& +O\left(T^{1+k\left(l-\frac{1}{4}\right)+\varepsilon} y^{-1 / 2}+T^{1 / 2+k\left(l-\frac{1}{4}\right)+\varepsilon} y^{s(k)}\right) .
\end{aligned}
$$

\subsection{Upper bound of the integral $\int_{T}^{2 T} F_{1}^{k-1}(x) F_{2}(x) d x$}

In this subsection we shall estimate the integral $\int_{T}^{2 T} F_{1}^{k-1}(x) F_{2}(x) d x$.

Let

$$
N_{2}:=H^{2}\left(2^{2 J+1}+1 / 2\right), J:=[(\mathcal{L}-\log \mathcal{L}) / 2 \log 2] .
$$

By (6.14) we have

$$
\begin{aligned}
F_{1}^{k-1}(x) F_{2}(x)=\theta_{l}^{k} x^{k(l-1 / 4)} \sum_{y<n_{1} \leq N_{2}} \sum_{n_{2} \leq y} \cdots \sum_{n_{k} \leq y} \frac{\tau_{l}\left(n_{1} ; H, T\right) \tau_{l}\left(n_{2}\right) \cdots \tau_{l}\left(n_{k}\right)}{\left(n_{1} \cdots n_{k}\right)^{3 / 4}} \\
\quad \times \prod_{j=1}^{k} \cos \left(2 \pi \sqrt{n_{j} x}-\frac{\pi}{4}\right) \\
=\frac{\theta_{l}^{k} x^{k(l-1 / 4)}}{2^{k-1}} \sum_{\mathbf{i} \in \mathbb{I}^{k-1}} \sum_{y<n_{1} \leq N_{2}} \sum_{n_{2} \leq y} \cdots \sum_{n_{k} \leq y} \frac{\tau_{l}\left(n_{1} ; H, T\right) \tau_{l}\left(n_{2}\right) \cdots \tau_{l}\left(n_{k}\right)}{\left(n_{1} \cdots n_{k}\right)^{3 / 4}} \\
\quad \times \cos \left(2 \pi \sqrt{x} \alpha(\mathbf{n} ; \mathbf{i})-\frac{\pi}{4} \beta(\mathbf{i})\right) .
\end{aligned}
$$

Thus

$$
F_{1}^{k-1}(x) F_{2}(x)=\frac{\theta_{l}^{k}}{2^{k-1}}\left(S_{3}(x)+S_{4}(x)\right)
$$

where

$$
\begin{gathered}
S_{3}(x)=x^{k(l-1 / 4)} \sum_{\mathbf{i} \in \mathbb{I}^{k-1}} \cos \left(-\frac{\pi \beta(\mathbf{i})}{4}\right) \sum_{\substack{y<n_{1} \leq N_{2} \\
S_{j} \leq y, 2 \leq j \leq k \\
\alpha(\mathbf{n} ; \mathbf{i}=0}} \frac{\tau_{l}\left(n_{1} ; H, T\right) \tau_{l}\left(n_{2}\right) \cdots \tau_{l}\left(n_{k}\right)}{\left(n_{1} \cdots n_{k}\right)^{3 / 4}}, \\
\sum_{4}(x)=x^{k(l-1 / 4)} \sum_{\substack{\mathbf{i} \in \mathbb{I}^{k-1} \\
y<n_{1} \leq N_{2}}} \sum_{\substack{n_{j} \leq y, 2 \leq j \leq k \\
\alpha(\mathbf{n} ; \mathbf{i}) \neq 0}} \frac{\tau_{l}\left(n_{1} ; H, T\right) \tau_{l}\left(n_{2}\right) \cdots \tau_{l}\left(n_{k}\right)}{\left(n_{1} \cdots n_{k}\right)^{3 / 4}} \\
\times \cos \left(2 \pi \alpha(\mathbf{n} ; \mathbf{i}) \sqrt{x}-\frac{\pi \beta(\mathbf{i})}{4}\right) .
\end{gathered}
$$


By Lemma 4.4 we have

$$
\begin{aligned}
& \int_{T}^{2 T} S_{3}(x) d x \\
& \ll \sum_{\mathbf{i} \in \mathbb{I}^{k-1}} \sum_{y<n_{1} \leq N_{2}} \sum_{\substack{n_{j} \leq y, 2 \leq j \leq k \\
\alpha(\mathbf{n} ; \mathbf{i})=0}} \frac{d\left(n_{1}\right) d\left(n_{2}\right) \cdots d\left(n_{k}\right)}{\left(n_{1} \cdots n_{k}\right)^{3 / 4}} \int_{T}^{2 T} x^{k(l-1 / 4)} d x
\end{aligned}
$$

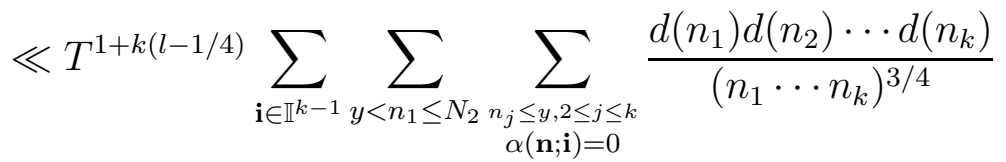

$$
\begin{aligned}
& \ll T^{1+k(l-1 / 4)} \sum_{v=1}^{k-1}\left|s_{k ; v}(d ; y)-s_{k ; v}(d)\right| \ll T^{1+k(l-1 / 4)+\varepsilon} y^{-1 / 2} .
\end{aligned}
$$

Now we consider the contribution of $S_{4}(x)$. By Lemma 4.5 we get

$$
\begin{aligned}
& \int_{T}^{2 T} S_{4}(x) d x \ll T^{1 / 2+k(l-1 / 4)}\left(\Sigma_{4}+\Sigma_{5}\right), \\
& \Sigma_{4}=\sum_{\mathbf{i} \in \mathbb{I}^{k-1}} \sum_{y<n_{1} \leq k^{2} y} \sum_{\substack{n_{j} \leq y, 2 \leq j \leq k \\
\alpha(\mathbf{n} ; \mathbf{i}) \neq 0}} \frac{d\left(n_{1}\right) \cdots d\left(n_{k}\right)}{\left(n_{1} \cdots n_{k}\right)^{3 / 4}|\alpha(\mathbf{n} ; \mathbf{i})|}, \\
& \Sigma_{5}=\sum_{\mathbf{i} \in \mathbb{I}^{k-1}} \sum_{k^{2}} \sum_{y<n_{1} \leq N_{2}} \sum_{\substack{n_{j} \leq y, 2 \leq j \leq k \\
\alpha(\mathbf{n} ; \mathbf{i}) \neq 0}} \frac{d\left(n_{1}\right) \cdots d\left(n_{k}\right)}{\left(n_{1} \cdots n_{k}\right)^{3 / 4}|\alpha(\mathbf{n} ; \mathbf{i})|} .
\end{aligned}
$$

By (6.21) we have

$$
\Sigma_{4} \ll U_{k}\left(k^{2} y\right) \ll y^{s(k)+\varepsilon} .
$$

When $n_{1}>k^{2} y$, it is easy to show that $|\alpha(\mathbf{n} ; \mathbf{i})| \gg n_{1}^{1 / 2}$, which implies that

$$
\Sigma_{5} \ll \sum_{k^{2} y<n_{1} \leq N_{2}} \sum_{n_{j} \leq y, 2 \leq j \leq k} \frac{d\left(n_{1}\right) \cdots d\left(n_{k}\right)}{\left(n_{2} \cdots n_{k}\right)^{3 / 4} n_{1}^{5 / 4}} \ll y^{\frac{k-2}{4}} \mathcal{L}^{k}
$$

if noting that

$$
\sum_{n \leq y} d(n) n^{-3 / 4} \ll y^{1 / 4} \log y, \quad \sum_{n>y} d(n) n^{-5 / 4} \ll y^{-1 / 4} \log y .
$$

From (6.24)-(6.28) we have

$$
\int_{T}^{2 T} F_{1}^{k-1}(x) F_{2}(x) d x \ll T^{1+k(l-1 / 4)+\varepsilon} y^{-1 / 2}+T^{1 / 2+k(l-1 / 4)+\varepsilon} y^{s(k)}
$$




\subsection{Higher-power moments of $F_{2}(x)$}

In this subsection we shall study the higher power moments of $F_{2}(x)$. From now on, we take $H:=T^{A_{0}}$.

We first study the mean-square of $F_{2}(x)$. Recall that $N_{2}=H^{2}\left(2^{2 J+1}+1 / 2\right)$. Since $\tau(n, H ; T)=\tau(n)$ for $n \leq y \leq T \mathcal{L}^{-2}$, we have

$$
\begin{aligned}
F_{2}(x) & =\theta_{l} x^{l-1 / 4} \sum_{y<n \leq N_{2}} \frac{\tau_{l}(n ; H, T)}{n^{3 / 4}} \cos (2 \pi \sqrt{n x}-\pi / 4)+O\left(T^{l-1 / 2} \mathcal{L}^{3}\right) \\
& \ll T^{l-1 / 4}\left|\sum_{y<n \leq N_{2}} \frac{\tau_{l}(n ; H, T)}{n^{3 / 4}} e(2 \sqrt{n x})\right|+T^{l-1 / 2} \mathcal{L}^{3},
\end{aligned}
$$

which implies

$$
\begin{aligned}
\int_{T}^{2 T} & F_{2}^{2}(x) d x \ll T^{2 l-1 / 2} \int_{T}^{2 T}\left|\sum_{y<n \leq N_{2}} \frac{\tau(n ; H, T)}{n^{3 / 4}} e(2 \sqrt{n x})\right|^{2} d x+T^{2 l} \mathcal{L}^{3} \\
& \ll T^{2 l+1 / 2} \sum_{y<n \leq N_{2}} \frac{d^{2}(n)}{n^{3 / 2}}+T^{2 l} \sum_{y<m<n \leq N_{2}} \frac{d(n) d(m)}{(m n)^{3 / 4}(\sqrt{n}-\sqrt{m})} \\
& \ll \frac{T^{2 l+1 / 2} \mathcal{L}^{3}}{y^{1 / 2}},
\end{aligned}
$$

where we used the estimate $\sum_{n \leq u} d^{2}(n) \ll u \log ^{3} u$ and the well-known Hilbert's inequality.

Now suppose $y$ satisfies $y^{2 b\left(K_{0}\right)} \leq T$. Hence from Lemma 6.2 we get that

$$
\int_{T}^{2 T}\left|F_{1}(x)\right|^{K_{0}} d x \ll T^{1+K_{0}(l-1 / 4)+\varepsilon},
$$

which implies

$$
\int_{T}^{2 T}\left|F_{1}(x)\right|^{A_{0}} d x \ll T^{1+A_{0}(l-1 / 4)+\varepsilon}
$$

since $A_{0} \leq K_{0}$. Trivially we have

$$
G(x)=\sum_{m \leq \sqrt{2 T}} \min \left(1, \frac{1}{H\left\|\frac{x}{2 m}-\frac{m}{2}+\frac{1}{2}\right\|}\right) \ll T^{1 / 2} .
$$


By Lemma 6.1 we have

$$
\int_{T}^{2 T} G^{A_{0}}(x) d x \ll T^{\left(A_{0}-1\right) / 2} \int_{T}^{2 T} G(x) d x \ll T^{A_{0} / 2+1} H^{-1} \mathcal{L} \ll T \mathcal{L} .
$$

From (2.3), (6.31) and (6.32) we get

$$
\int_{T}^{2 T}\left|F_{2}(x)\right|^{A_{0}} d x \ll \int_{T}^{2 T}\left(\left|R_{*}(x)\right|^{A_{0}}+\left|F_{1}(x)\right|^{A_{0}}+T^{A_{0}(l-1 / 2)} G^{A_{0}}(x)\right) d x \ll T^{1+A_{0}(l-1 / 4)+\varepsilon} .
$$

For any $2<A<A_{0}$, from (6.30), (6.33) and Hölder's inequality we get that

$$
\begin{gathered}
\int_{T}^{2 T}\left|F_{2}(x)\right|^{A} d x=\int_{T}^{2 T}\left|F_{2}(x)\right|^{\frac{2\left(A_{0}-A\right)}{A_{0}-2}+\frac{A_{0}(A-2)}{A_{0}-2}} d x \\
\ll\left(\int_{T}^{2 T} F_{2}^{2} x d x\right)^{\frac{A_{0}-A}{A_{0}-2}}\left(\int_{T}^{2 T}\left|F_{2}(x)\right|^{A_{0}} d x\right)^{\frac{A-2}{A_{0}-2}} \ll T^{1+A\left(l-\frac{1}{4}\right)+\varepsilon} y^{-\frac{A_{0}-A}{2\left(A_{0}-2\right)}} .
\end{gathered}
$$

Namely, we have the following Lemma 6.3.

Lemma 6.3. Suppose $T^{\varepsilon} \leq y \leq T^{1 / 2 b\left(K_{0}\right)}, 2<A<A_{0}$, then

$$
\int_{T}^{2 T}\left|F_{2}(x)\right|^{A} d x \ll T^{1+A\left(l-\frac{1}{4}\right)+\varepsilon} y^{-\frac{A_{0}-A}{2\left(A_{0}-2\right)}} .
$$

\subsection{Evaluation of the integral $\int_{T}^{2 T} F^{k}(x) d x$.}

Suppose $3 \leq k<A_{0}$ and $T^{\varepsilon} \leq y \leq T^{1 / 2 b\left(K_{0}\right)}$. By the elementary formula

$$
(a+b)^{k}=a^{k}+k a^{k-1} b+O\left(\left|a^{k-2} b^{2}\right|+|b|^{k}\right)
$$

we get

$$
\begin{aligned}
\int_{T}^{2 T} F^{k}(x) d x & =\int_{T}^{2 T} F_{1}^{k} d x+k \int_{T}^{2 T} F_{1}^{k-1}(x) F_{2}(x) d x \\
& +O\left(\int_{T}^{2 T}\left|F_{1}^{k-2}(x) F_{2}^{2}(x)\right| d x+\int_{T}^{2 T}\left|F_{2}(x)\right|^{k} d x\right) .
\end{aligned}
$$

By (6.31), Lemma 6.3 and Hölder's inequality we get

$$
\begin{gathered}
\int_{T}^{2 T}\left|F_{1}^{k-2}(x) F_{2}^{2}(x)\right| d x \\
\ll\left(\int_{T}^{2 T}\left|F_{1}(x)\right|^{A_{0}} d x\right)^{\frac{k-2}{A_{0}}}\left(\int_{T}^{2 T}\left|F_{2}(x)\right|^{\frac{2 A_{0}}{A_{0}-k+2}} d x\right)^{\frac{A_{0}-k+2}{A_{0}}} \\
\ll T^{1+k\left(l-\frac{1}{4}\right)+\varepsilon} y^{-\frac{A_{0}-k}{2\left(A_{0}-2\right.}} .
\end{gathered}
$$


Now from (6.29), (6.37), Lemma 6.2 and Lemma $6.3(A=k)$ we get

$$
\begin{aligned}
& \int_{T}^{2 T} F^{k}(x) d x \\
= & \frac{2^{1+k-l k} l^{k} B_{k}\left(\tau_{l}\right)}{(l !)^{k} \pi^{k}} \int_{T}^{2 T} x^{k(l-1 / 4)} d x+O\left(T^{1+k(l-1 / 4)-\frac{A_{0}-k}{4\left(A_{0}-2\right) s\left(K_{0}\right)}+\varepsilon}\right) \\
= & \frac{2^{1+k-l k} l^{k} B_{k}\left(\tau_{l}\right)}{(l !)^{k}} \pi^{k} \int_{T}^{2 T} x^{k(l-1 / 4)} d x+O\left(T^{1+k(l-1 / 4)-\delta_{1}\left(k, A_{0}\right)+\varepsilon}\right)
\end{aligned}
$$

by choosing $y=T^{1 / 2 s\left(K_{0}\right)}$.

\subsection{Proof of Theorem 2}

By Proposition 6.1 and the elementary formula $(a+b)^{k}=a^{k}+O\left(|a|^{k-1}|b|+|b|^{k}\right)$ we get

$$
\begin{aligned}
R_{*}^{k}(x)=F^{k}(x)+O\left(|F(x)|^{k-1} T^{l-1 / 2} G(x)+|F(x)|^{k-1} T^{l-1 / 2} \mathcal{L}^{3}\right) \\
+O\left(T^{k(l-1 / 2)} G^{k}(x)+T^{k / 2} \mathcal{L}^{3 k}\right) .
\end{aligned}
$$

By Lemma 6.1 and Hölder's inequality we get

$$
\begin{aligned}
& \int_{T}^{2 T}|F(x)|^{k-1} T^{l-1 / 2} G(x) d x \\
& \ll T^{l-1 / 2}\left(\int_{T}^{2 T}|F(x)|^{A_{0}} d x\right)^{\frac{k-1}{A_{0}}}\left(\int_{T}^{2 T} G(x)^{\frac{A_{0}}{A_{0}-k+1}} d x\right)^{\frac{A_{0}-k+1}{A_{0}}} \\
& \ll T^{l-1 / 2} T^{\left(1+A_{0}\left(l-\frac{1}{4}\right)+\varepsilon\right) \frac{k-1}{A_{0}}}\left(T^{\frac{k-1}{2\left(A_{0}-k+1\right)}} \int_{T}^{2 T} G(x) d x\right)^{\frac{A_{0}-k+1}{A_{0}}} \\
& \ll T^{l-1 / 2} T^{\left(1+\frac{3 A_{0}}{4}+\varepsilon\right) \frac{k-1}{A_{0}}}\left(T^{\frac{k-1}{A_{0}-k+1}} T^{-\left(A_{0}-2\right)}\right)^{\frac{A_{0}-k+1}{A_{0}}} \\
& \ll T^{1 / 4+k(l-1 / 4)} .
\end{aligned}
$$

By Hölder's inequality again we get

$$
\begin{aligned}
& \int_{T}^{2 T}|F(x)|^{k-1} T^{l-1 / 2} \mathcal{L}^{3} d x \\
& \ll T^{l-1 / 2} \mathcal{L}^{3}\left(\int_{T}^{2 T}|F(x)|^{A_{0}} d x\right)^{\frac{k-1}{A_{0}}} T^{\frac{A_{0}-k+1}{A_{0}}} \\
& \ll T^{1+k(l-1 / 4)-1 / 4+\varepsilon} .
\end{aligned}
$$


From (6.39)-(6.41) we get

$$
\int_{T}^{2 T} R_{*}^{k}(x) d x=\frac{2^{1+k-l k} l^{k} B_{k}\left(\tau_{l}\right)}{(l !)^{k} \pi^{k}} \int_{T}^{2 T} x^{k\left(l-\frac{1}{4}\right)} d x+O\left(T^{1+k(l-1 / 4)-\delta_{1}\left(k, A_{0}\right)+\varepsilon}\right),
$$

which implies that

$$
\begin{aligned}
& \int_{1}^{T} R_{*}^{k}(x) d x \\
= & \frac{2^{1+k-l k} l^{k} B_{k}\left(\tau_{l}\right)}{(l !)^{k} \pi^{k}} \int_{1}^{T} x^{k\left(l-\frac{1}{4}\right)} d x+O\left(T^{1+k(l-1 / 4)-\delta_{1}\left(k, A_{0}\right)+\varepsilon}\right) \\
= & \frac{2^{1+k-l k} l^{k} B_{k}\left(\tau_{l}\right)}{(l !)^{k} \pi^{k}(k(l-1 / 4)+1)} T^{1+k(l-1 / 4)}+O\left(T^{1+k(l-1 / 4)-\delta_{1}\left(k, A_{0}\right)+\varepsilon}\right) .
\end{aligned}
$$

Now Theorem 2 follows from (6.42) if noting that

$$
\int_{1}^{T} R^{k}(t) d t=2 \pi \int_{1}^{\frac{T}{2 \pi}} R_{*}^{k}(x) d x+O(1) .
$$

\section{Proofs of Theorem 3 and Theorem 4}

In this section we prove Theorem 3 and Theorem 4 . Throughout this section, let $A_{0}=262 / 27$ and $H=T^{262 / 27}$.

\subsection{Proof of Theorem 3}

The proof of Theorem 3 is almost the same as that of Theorem 2. So we give only an outline.

Using the argument of the proof of Theorem 5.1 to $F_{1}(x)$ directly, we can show that the estimate

$$
\int_{T}^{2 T}\left|F_{1}(x)\right|^{A_{0}} d x \ll T^{1+A_{0}(l-1 / 4)} \mathcal{L}^{50}
$$

holds for $y \leq T^{77 / 208}$. Here we remark that if we want to get the result of the type (7.1) we have to assume $y T^{2 \theta} \ll T$ when recalling the formula (5.8), where $\theta=131 / 416$.

From (7.1), (6.32) and (2.3) we get

$$
\int_{T}^{2 T}\left|F_{2}(x)\right|^{A_{0}} d x \ll T^{1+A_{0}(l-1 / 4)+\varepsilon} .
$$


From (7.1) and (7.2) we can show that Lemma 6.2 holds for $y \leq T^{77 / 208}$. Using other estimates in the proof of Theorem 2 , we can get by choosing $y=$ $T^{(k-2) / 2\left(A_{0}-2\right) s(k)}$ that the estimate

$$
\begin{aligned}
& \int_{T}^{2 T} R_{*}^{k}(x) d x= \frac{2^{1+k-l k} l^{k} B_{k}\left(\tau_{l}\right)}{(l !)^{k} \pi^{k}} \int_{T}^{2 T} x^{k\left(l-\frac{1}{4}\right)} d x+O\left(T^{1+k\left(l-\frac{1}{4}\right)+\varepsilon} y^{-\frac{A_{0}-k}{2\left(A_{0}-2\right)}}\right) \\
&+O\left(T^{1 / 2+k(l-1 / 4)+\varepsilon} y^{s(k)}\right) \\
&=\frac{2^{1+k-l k} l^{k} B_{k}\left(\tau_{l}\right)}{(l !)^{k} \pi^{k}} \int_{T}^{2 T} x^{k\left(l-\frac{1}{4}\right)} d x+O\left(T^{1+k(l-1 / 4)-\delta_{2}(k, 262 / 27)+\varepsilon}\right)
\end{aligned}
$$

holds, which implies Theorem 3.

\subsection{Proof of Theorem $4($ the case $k=3)$}

By the argument of Section 6.3 we get

$$
\begin{aligned}
\int_{T}^{2 T} F_{1}^{3}(x) d x & =\frac{2^{4-2 l} l^{3} B_{3}\left(\tau_{l}\right)}{(l !)^{3} \pi^{3}} \int_{T}^{2 T} x^{3\left(l-\frac{1}{4}\right)} d x \\
& +O\left(T^{3 l+1 / 4+\varepsilon} y^{-1 / 2}\right)+O\left(T^{3 l-1 / 4} U_{3}(y)\right)
\end{aligned}
$$

where $U_{3}(y)$ was defined in Section 6.3. In Lemma 2.6 of [32], the author proved

$$
U_{3}(y) \ll y^{1 / 4+\varepsilon} .
$$

By the argument of Section 6.4 we get

$$
\int_{T}^{2 T} F_{1}^{2}(x) F_{2}(x) d x \ll T^{3 l+1 / 4+\varepsilon} y^{-1 / 2}+T^{3 l-1 / 4} y^{\frac{1}{4}} \mathcal{L}^{3}+T^{3 l-1 / 4} U_{3}^{*}(y),
$$

where

$$
U_{3}^{*}(y)=\sum_{\mathbf{i} \in \mathbb{I}^{2}} \sum_{y<n_{1} \leq 9} \sum_{\substack{n_{2} \leq y, n_{3} \leq y \\ \alpha(\mathbf{n} ; \mathbf{i}) \neq 0}} \frac{d\left(n_{1}\right) d\left(n_{2}\right) d\left(n_{3}\right)}{\left(n_{1} n_{2} n_{3}\right)^{3 / 4}|\alpha(\mathbf{n} ; \mathbf{i})|} .
$$

By (7.4) we get

$$
U_{3}^{*} y \ll U_{3}(9 y) \ll y^{1 / 4+\varepsilon} .
$$

Trivially we have $F_{1}(x) \ll x^{l-1 / 4} y^{1 / 4} \mathcal{L}$. So by $(6.30)$ we get that

$$
\int_{T}^{2 T}\left|F_{1}(x) F_{2}^{2}(x)\right| d x \ll T^{l-1 / 4} y^{1 / 4} \mathcal{L} \int_{T}^{2 T}\left|F_{2}^{2}(x)\right| d x \ll T^{3 l+1 / 4} y^{-1 / 4} \mathcal{L}^{4}
$$


holds for $y \leq T$. By the trivial estimate $F_{2}(x) \ll x^{l}$ and (6.30) we get

$$
\int_{T}^{2 T}\left|F_{2}^{3}(x)\right| d x \ll T^{l} \int_{T}^{2 T}\left|F_{2}(x)\right|^{2} d x \ll T^{3 l+1 / 2} y^{-1 / 2} \mathcal{L}^{3} .
$$

From (6.36) with $k=3$ and (7.3)-(7.8) with $y=T \mathcal{L}^{-2}$ we get

$$
\int_{T}^{2 T} F^{3}(x) d x=\frac{2^{4-2 l} l^{3} B_{3}\left(\tau_{l}\right)}{(l !)^{3} \pi^{3}} \int_{T}^{2 T} x^{3(l-1 / 4)} d x+O\left(T^{3 l+\varepsilon}\right),
$$

which combining the arguments of Section 6.7 gives

$$
\int_{T}^{2 T} R_{*}^{3}(x) d x=\frac{2^{4-2 l} l^{3} B_{3}\left(\tau_{l}\right)}{(l !)^{3} \pi^{3}} \int_{T}^{2 T} x^{3(l-1 / 4)} d x+O\left(T^{3 l+\varepsilon}\right) .
$$

The formula (2.8) follows from (7.9).

\subsection{Proof of Theorem $4($ the case $k=4)$}

By the argument of Section 6.3 we get

$$
\begin{aligned}
\int_{T}^{2 T} F_{1}^{4}(x) d x & =\frac{2^{5-4 l} l^{4} B_{4}\left(\tau_{l}\right)}{(l !)^{4} \pi^{4}} \int_{T}^{2 T} x^{4 l-1} d x \\
& +O\left(T^{4 l+\varepsilon} y^{-1 / 2}\right)+O\left(T^{4 l-1} V_{1,4}(y)\right)
\end{aligned}
$$

where

$$
V_{1,4}(y)=\sum_{\mathbf{i} \in \mathbb{I}^{3}} \sum_{\substack{n_{j} \leq y, 1 \leq j \leq 4 \\ \alpha(\mathbf{n} ; \mathbf{i}) \neq 0}} \frac{d\left(n_{1}\right) d\left(n_{2}\right) d\left(n_{3}\right) d\left(n_{4}\right)}{\left(n_{1} n_{2} n_{3} n_{4}\right)^{3 / 4}} \min \left(T, \frac{T^{1 / 2}}{|\alpha(\mathbf{n} ; \mathbf{i})|}\right) .
$$

In [31] the author proved that if $y \ll T^{3 / 4}$, then

$$
V_{1,4}(y) \ll T^{1-3 / 28+\varepsilon} .
$$

From (7.10) and (7.11) we get

$$
\int_{T}^{2 T} F_{1}^{4}(x) d x=\frac{2^{5-4 l} l^{4} B_{4}\left(\tau_{l}\right)}{(l !)^{4} \pi^{4}} \int_{T}^{2 T} x^{4 l-1} d x+O\left(T^{4 l-3 / 28+\varepsilon}\right) .
$$

By the argument of Section 6.4 we get

$$
\int_{T}^{2 T} F_{1}^{3}(x) F_{2}(x) d x \ll T^{4 l+\varepsilon} y^{-1 / 2}+T^{4 l-1 / 2} y^{1 / 2} \mathcal{L}^{4}+T^{4 l-1} V_{2,4}(y),
$$


where

$$
V_{2,4}(y)=\sum_{\mathbf{i} \in \mathbb{I}^{3}} \sum_{y<n_{1} \leq 16} \sum_{\substack{n_{j} \leq y, 2 \leq j \leq 4 \\ \alpha(\mathbf{n} ; \mathbf{i}) \neq 0}} \frac{d\left(n_{1}\right) d\left(n_{2}\right) d\left(n_{3}\right) d\left(n_{4}\right)}{\left(n_{1} n_{2} n_{3} n_{4}\right)^{3 / 4}} \min \left(T, \frac{T^{1 / 2}}{|\alpha(\mathbf{n} ; \mathbf{i})|}\right) .
$$

From (7.11) we get that if $y \ll T^{3 / 4}$, then

$$
V_{2,4}(y) \ll V_{1,4}(16 y) \ll T^{1-3 / 28+\varepsilon} .
$$

From Section 7.1 we know that Lemma 6.3 holds for $y \leq T^{77 / 208}$. Taking $A=4$ in Lemma 6.3 we get

$$
\int_{T}^{2 T} F_{2}^{4}(x) d x \ll T^{4 l+\varepsilon} y^{-77 / 208}
$$

Taking $k=4$ in (6.37) we get

$$
\int_{T}^{2 T} F_{1}^{2}(x) F_{2}^{2}(x) d x \ll T^{4 l+\varepsilon} y^{-77 / 208}
$$

holds for $y \leq T^{77 / 208}$.

From (7.10)-(7.16) and taking $y=T^{1 / 3}$ we get

$$
\int_{T}^{2 T} F^{4}(x) d x=\frac{2^{5-4 l} l^{4} B_{4}\left(\tau_{l}\right)}{(l !)^{4} \pi^{4}} \int_{T}^{2 T} x^{4 l-1} d x+O\left(T^{4 l-3 / 28+\varepsilon}\right),
$$

which combining the arguments of Section 6.7 gives

$$
\int_{T}^{2 T} R_{*}^{4}(x) d x=\frac{2^{5-4 l} l^{4} B_{4}\left(\tau_{l}\right)}{(l !)^{4} \pi^{4}} \int_{T}^{2 T} x^{4 l-1} d x+O\left(T^{4 l-3 / 28+\varepsilon}\right) .
$$

The formula (2.9) follows from (7.17).

\section{Proofs of Theorem 5 and Theorem 6}

We shall follow Heath-Brown's argument 11] to prove Theorem 5 and Theorem 6 . We first quote some results from [11. The following Hypothesis(H), Lemma 8.1 and Lemma 8.2 are Hypothesis(H), Theorem 5 and Theorem 6 of [11], respectively. 
Hypothesis(H): Let $M(t)$ be a real valued function, $a_{1}(t), a_{2}(t), \cdots$, be continuous real valued function with period 1 , and suppose there are non-zero constants $\gamma_{1}, \gamma_{2}, \cdots$ such that

$$
\lim _{N \rightarrow \infty} \limsup _{T \rightarrow \infty} \frac{1}{T} \int_{0}^{T} \min \left(1,\left|M(t)-\sum_{n \leq N} a_{n}\left(\gamma_{n} t\right)\right|\right) d t=0 .
$$

Lemma 8.1. Suppose $M(t)$ satisfies $(H)$ and suppose that the constants $\gamma_{i}$ are linearly independently over $\mathbb{Q}$. Suppose further

$$
\begin{aligned}
& \int_{0}^{1} a_{n}(t) d t=0 \quad(n \in \mathbb{N}) \\
& \sum_{n=1}^{\infty} \int_{0}^{1} a_{n}^{2}(t) d t<\infty
\end{aligned}
$$

and there is a constant $\mu>1$ such that

$$
\begin{aligned}
& \max _{t \in[0,1]}\left|a_{n}(t)\right| \ll n^{1-\mu}, \\
& \lim _{n \rightarrow \infty} n^{\mu} \int_{0}^{1} a_{n}^{2}(t) d t=\infty .
\end{aligned}
$$

Then $M(t)$ has a distribution function $f(\alpha)$ with the properties described in Theorem 5 .

Lemma 8.2. Suppose $M(t)$ satisfies $(\mathrm{H})$ and that

$$
\int_{0}^{T}|M(t)|^{K} d t \ll T
$$

holds for some positive number $K$. Then for any real number $k \in[0, K)$, the limit

$$
\lim _{T \rightarrow \infty} \frac{1}{T} \int_{0}^{T}|M(t)|^{K} d t
$$

exists.

Suppose $T \leq x \leq 2 T, H=T^{2}, J=[(\mathcal{L}-\log \mathcal{L}) / 2 \log 2]$. Define

$$
\begin{aligned}
M(x) & =x^{-(2 l-1 / 2)} R_{*}\left(x^{2}\right), \\
a_{n}(x) & =\frac{\mu^{2}(n)}{n^{3 / 4}} \sum_{r=1}^{\infty} \frac{\tau_{l}\left(n r^{2}\right)}{r^{3 / 2}} \cos \left(2 \pi r x-\frac{\pi}{4}\right), \\
\gamma_{n} & =\sqrt{n} .
\end{aligned}
$$


It is easy to check that $a_{n}(x)$ satisfies all conditions of Lemma 8.1 for any fixed constant $3 / 2<\mu<7 / 4$. By Proposition 6.1 we have

$$
\begin{aligned}
& M(x)=M_{1}(x)+M_{2}(x)+M_{3}(x), \\
& M_{1}(x)=\frac{2^{2-l}}{(l-1) ! \pi} \sum_{1 \leq n \leq T \mathcal{L}^{-2}} \frac{\tau_{l}(n)}{n^{3 / 4}} \cos \left(2 \pi x \sqrt{n}-\frac{\pi}{4}\right), \\
& M_{2}(x)=\frac{2^{2-l}}{(l-1) ! \pi} \sum_{T \mathcal{L}^{-2}<n \leq H^{2}\left(2^{2 J+1}+1 / 2\right)} \frac{\tau_{l}(n ; H, T)}{n^{3 / 4}} \cos \left(2 \pi x \sqrt{n}-\frac{\pi}{4}\right), \\
& M_{3}(x)=O\left(T^{-1 / 2} G\left(x^{2}\right)+T^{-1 / 2} \mathcal{L}^{3}\right) .
\end{aligned}
$$
$d(n))$

$$
\begin{gathered}
\left|M(x)-\sum_{n \leq N} a_{n}\left(\gamma_{n} x\right)\right| \\
\ll\left|\sum_{n \leq T \mathcal{L}^{-2}}^{\prime} \frac{\tau_{l}(n)}{n^{3 / 4}} \cos \left(2 \pi x \sqrt{n}-\frac{\pi}{4}\right)\right|+\sum_{n \leq N} \frac{1}{n^{3 / 4}} \sum_{r>\sqrt{T} / \sqrt{n}} \frac{d\left(n r^{2}\right)}{r^{3 / 2}}+\left|M_{2}(x)\right|+M_{3}(x) \\
\ll\left|\sum_{n \leq T \mathcal{L}^{-2}}^{\prime} \frac{\tau_{l}(n)}{n^{3 / 4}} \cos \left(2 \pi x \sqrt{n}-\frac{\pi}{4}\right)\right|+N^{1 / 2} T^{-1 / 4} \mathcal{L}^{5}+\left|M_{2}(x)\right|+M_{3}(x),
\end{gathered}
$$

where $\sum^{\prime}$ means that $n$ has a square-free kernel great that $N$.

We have(note that $\left.\tau_{l}(n) \ll d(n)\right)$

$$
\begin{aligned}
\int_{T}^{2 T}\left|\sum_{n \leq T \mathcal{L}^{-2}}^{\prime} \frac{\tau_{l}(n)}{n^{3 / 4}} \cos \left(2 \pi x \sqrt{n}-\frac{\pi}{4}\right)\right|^{2} d x & =T \sum_{n \leq T \mathcal{L}^{-2}}^{\prime} \frac{d^{2}(n)}{n^{3 / 2}}+O\left(T^{\varepsilon}\right) \\
& \ll T \sum_{n>N} \frac{d^{2}(n)}{n^{3 / 2}}+T^{\varepsilon} \\
& \ll T N^{-1 / 2} \log ^{3} N+T^{\varepsilon}
\end{aligned}
$$

Similar to (6.30) we have

$$
\int_{T}^{2 T}\left|M_{2}(x)\right|^{2} d x \ll T N^{-1 / 2} \log ^{3} N .
$$


By the trivial estimate $G(u) \ll \sqrt{u}(u \sim T)$ and Lemma 6.1 we get

$$
\begin{aligned}
& \int_{T}^{2 T}\left|M_{3}(x)\right|^{2} d x \ll T^{-1} \int_{T}^{2 T} G^{2}\left(x^{2}\right) d x+\mathcal{L}^{6} \\
& \ll \int_{T}^{2 T} G\left(x^{2}\right) d x+\mathcal{L}^{6} \ll \int_{\sqrt{T}}^{\sqrt{2 T}} G(y) y^{-1 / 2} d y+\mathcal{L}^{6} \\
& \ll T^{1 / 2} H^{-1} \mathcal{L}+\mathcal{L}^{6} \ll \mathcal{L}^{6} .
\end{aligned}
$$

From the above estimates and Cauchy's inequality we get

$$
\limsup _{T \rightarrow \infty} \frac{1}{T} \int_{T}^{2 T}\left|M(x)-\sum_{n \leq N} a_{n}\left(\gamma_{n} x\right)\right|^{2} d x \ll N^{-1 / 2} \log ^{3} N
$$

and whence Hypothesis $(\mathrm{H})$ follows. From Lemma 8.1 with $\mu=5 / 3$ we get Theorem 5 .

Now we prove Theorem 6. According to Lemma 8.2 , if we can show that the estimate

$$
\int_{0}^{T} x^{-k(l-1 / 4)}|R(x)|^{k} d x \ll T
$$

holds for any real number $0 \leq k<A_{0}:=262 / 27$, then Theorem 6 follows directly.

Suppose $2<k<A_{0}$ is fixed. Suppose $x \sim T, y=T^{1 / s(10)}, H=T^{5}$. We have

$$
R(x) \ll\left|F_{1}(x)\right|+\left|F_{2}(x)\right|+T^{l-1 / 2} G(x)+T^{l-1 / 2} \mathcal{L}^{3},
$$

where $F_{1}(x), F_{2}(x)$ and $G(x)$ were defined in Section 6 . By Lemma 6.2 we get

$$
\int_{T}^{2 T}\left|F_{1}(x)\right|^{10} d x \ll T^{1+10(l-1 / 4)},
$$

which implies that

$$
\int_{T}^{2 T}\left|F_{1}(x)\right|^{k} d x \ll T^{1+k(l-1 / 4)}
$$

By Lemma 6.3 we get

$$
\int_{T}^{2 T}\left|F_{2}(x)\right|^{k} d x \ll T^{1+k(l-1 / 4)-\left(A_{0}-k\right) / 2\left(A_{0}-2\right) s(10)+\varepsilon} \ll T^{1+k(l-1 / 4)} .
$$


By Lemma 6.1 we get

$$
\int_{T}^{2 T} T^{k(l-1 / 2)} G^{k}(x) d x \ll T^{k(l-1 / 2)} T^{(k-1) / 2} \int_{T}^{2 T} G(x) d x \ll T^{k(l-1 / 4)-2} .
$$

From the above estimates we get that

$$
\int_{T}^{2 T}|R(x)|^{k} d x \ll T^{1+k(l-1 / 4)}
$$

and correspondingly,

$$
\int_{0}^{T}|R(x)|^{k} d x \ll T^{1+k(l-1 / 4)}
$$

which implies (8.1) by partial summation. This completes the proof of Theorem 6 .

\section{References}

[1] V. Bentkus, F. Götze, Lattice point problems and distribution of values of quadratic forms, Ann. of Math. (2)50: 3(1999), 977-1027.

[2] P. H. Bérard, On the wave equation on a compact Riemannian manifold without conjugate points, Math. Z. 155:3(1977), 249-276.

[3] L. Bleher, On the distribution of the number of lattice points inside a family of convex ovals, Duke Math. J. 67: 3(1992), 461-481.

[4] D. Chung, Y. N. Petridis and J. Toth, The remainder in Weyl's law for Heisenberg manifolds II, Bonner Mathematische Schriften, Nr. 360, Bonn, 2003, 16 pages.

[5] H. Cramér, Über zwei Sätze von Herrn G. H. Hardy, Math. Z.15(1922), 201-210.

[6] G. B. Folland, Harmonic Analysis in Phase Space, Princeton University Press(1989), 9-73.

[7] F. Fricker, Einführung in die Gitterpunketlehre, [Introduction to lattice point theory] Lehrbücher und Monographien aus dem Gebiete der Exakten Wissenschaften(LMW), Mathematische Reihe[Textbooks and Monographs in the Exact Sciences]73, Birkhäuser Verlag, Basel-Boston, Mas., 1982. 
[8] C. Gordon, E. Wilson, The spectrum of the Laplacian on Riemannian Heisenberg manifolds, Michigan Math. J. 33(2) (1986), 253-271.

[9] F. Götze, Lattice point problems and values of quadratic forms, Inventiones Mathematicae157(2004), 195-226.

[10] G. H. Hardy, On the expression of a number as the sum of two squares, Quart. J Math. 46(1915), 263-283.

[11] D. R. Heath-Brown, The distribution and moments of the error term in the Dirichlet divisor problem, Acta Arith. (1992), 389-415.

[12] D. R. Heath-Brown, The Piatetski-Shapiro prime theorem, J.of Number theory, Vol16(1983), 242-266.

[13] L. Hörmander, The spectral function of an elliptic operator, Acta Math.121(1968), 193-218.

[14] M. N. Huxley, Exponential sums and lattice points III, Proc. London Math. Soc. 87 (3) (2003), 591-609.

[15] A. Ivić, The Riemann-zeta function, John Wiley \& Sons, New York, 1985.

[16] V.YA. Ivrii, Precise Spectral Asymptotics for elliptic Operators Acting in Fibrings over Manifolds with Boundary, Springer Lecture notes in Mathematics 1100(1984).

[17] M. Khosravi, Third moment of the remainder error term in Weyl's law for Heisenberg manifolds, arXiv: 0711.0073.

[18] M. Khosravi, Y. Petridis, The remainder in Weyl's law for $n$-dimensional Heisenberg manifolds, Proc. of the American Math. Soc. 133(2005), 3561-3571.

[19] M. Khosravi, J. Toth, Cramér's formula for Heisenberg manifolds, Ann. de l'institut Fourier 55(2005), 2489-2520.

[20] M. Kuhleitner, W. G. Nowak, The asymptotic behaviour of the mean-square of fractional part sums, Proc. Edinb. Math. Soc. 43(2000), 309-323.

[21] S. H. Min, The methods of number theory(in Chinese), Science Press, Beijing: 1981.

[22] W. Müller, On the asymptotic behaviour of the ideal counting function in quadratic number fields, Monatsh. Math. 108 (1989), no. 4, 301-323. 
[23] W. G. Nowak, Fractional part sums and lattice points, Proc. Edinb. Math. Soc.41(1998), 497-515.

[24] Y. Petribis, J. Toth, The remainder in Weyl's law for Heisenberg manifolds, J. Differential Geom. 60(2002), 455-483.

[25] E. M. Stein, Harmonic Analysis, Princeston University Press(1993), 527-574.

[26] Kai-Man Tsang, Higher-power moments of $\Delta(x), E(t)$ and $P(x)$, Proc. London Math. Soc.(3)65(1992), 65-84.

[27] J. D. Vaaler, Some extremal functions in Fourier analysis, Bull. Amer. Math. Soc. 12 (1985), 183-216.

[28] I. M. Vinogradov, Special variants of the method of trigonometric sums, (Nauka, Moscow), 1976; English transl. in his Selected works (Springer-Verlag), 1985.

[29] A. V. Volovoy, Improved two-term asymptotics for the eigenvalue distribution function of an elliptic operator on a compact manifold, Comm. Partial Differemtial Equations15: 11(1990), 1509-1563.

[30] Wenguang Zhai, On higher-power moments of $\Delta(x)$ (II), Acta Arith. 114 (2004), 35-54.

[31] Wenguang Zhai, On higher-power moments of $\Delta(x)$ (III), Acta Arith. 118 (2005), 263-281.

[32] Wenguang Zhai, On higher-power moments of E(t), Acta Arith. Vol.115(2004), 329-348.

Wenguang Zhai,

School of Mathematical Sciences,

Shandong Normal University,

Jinan, Shandong, 250014,

P.R.China

E-mail:zhaiwg@hotmail.com 